ACCepted to Ap.J. April 23, 2007

Preprint typeset using $\mathrm{L}_{\mathrm{T}}^{\mathrm{T}} \mathrm{X}$ style emulateapj v. 08/22/09

\title{
SIMULATIONS OF MAGNETICALLY-DRIVEN SUPERNOVA AND HYPERNOVA EXPLOSIONS IN THE CONTEXT OF RAPID ROTATION
}

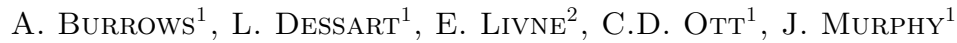 \\ Accepted to Ap.J. April 23, 2007
}

\begin{abstract}
We present here the first 2D rotating, multi-group, radiation magnetohydrodynamics (RMHD) simulations of supernova core collapse, bounce, and explosion. In the context of rapid rotation, we focus on the dynamical effects of magnetic stresses and the creation and propagation of MHD jets. We find that a quasi-steady state can be quickly established after bounce, during which a well-collimated MHD jet is maintained by continuous pumping of power from the differentially rotating core. If the initial spin period of the progenitor core is $\lesssim 2$ seconds, the free energy reservoir in the secularly evolving protoneutron star is adequate to power a supernova explosion, and may be enough for a hypernova. The jets are well collimated by the infalling material and magnetic hoop stresses, and maintain a small opening angle. We see evidence of sausage instabilities in the emerging jet stream. Neutrino heating is sub-dominant in the rapidly rotating models we explore, but can contribute $10-25 \%$ to the final explosion energy. Our simulations suggest that even in the case of modest or slow rotation, a supernova explosion might be followed by a secondary, weak MHD jet explosion, which, because of its weakness may to date have gone unnoticed in supernova debris. Furthermore, we suggest that the generation of a non-relativistic MHD precursor jet during the early protoneutron star/supernova phase is implicit in both the collapsar and "millisecond magnetar" models of GRBs. The multi-D, multi-group, rapidly rotating RMHD simulations we describe here are a start along the path towards more realistic simulations of the possible role of magnetic fields in some of Nature's most dramatic events.

Subject headings: supernovae, multi-dimensional radiation magneto-hydrodynamics, MHD
\end{abstract}

\section{INTRODUCTION}

There is an emerging consensus that the core-collapse supernova mechanism is essentially aspherical and that instabilities and the breaking of spherical symmetry are keys to explosion (Buras et al. 2006ab; Burrows et al. 2006,2007; Blondin, Mezzacappa, \& DeMarino 2003). In modern simulations, due to copious neutrino losses, the tamp of accretion, and photodissociation, the direct hydrodynamic bounce mechanism always fails. The delayed neutrino-driven mechanism works in spherical models only for a small subset of the lightest massive star progenitors (e.g., $8.8 \mathrm{M}_{\odot}$; Kitaura, Janka, \& Hillebrandt 2006). It works, if only marginally, in 2D simulations when aided by post-shock convection and overturn only for slightly more massive progenitors (e.g, 11.2 $\mathrm{M}_{\odot}$; Buras et al. 2006b). The neutrino mechanism does work in the context of accretion-induced collapse (AIC; Dessart et al. 2006a), but these systems must be rare. In all cases explored to date, when neutrino heating is the driving agency the explosion energies are not the canonical supernova values near $10^{51}$ ergs (三 one Bethe), but half a dex or more smaller. The low accretion densities that enable explosion also inhibit the deposition of neutrino energy. However, it is indeed possible that when credible 3D simulations are available, we will find that a robust neutrino mechanism obtains for the majority of the relevant progenitor mass range $\left(8<M<50\right.$ ? $\left.\mathrm{M}_{\odot}\right)$.

Recently, Burrows et al. $(2006,2007)$ have found that if the delay to explosion is long, perhaps 0.5 to 1.0 seconds, the inner core can be excited to oscillate in an $\ell=1 \mathrm{~g}$-mode which damps by the anisotropic emission of sound. At this late stage, acoustic power can rival or exceed neutrino deposition power and can explode the envelope. The excitation of core g-modes in this manner is unavoidable. The question is whether the pulsation amplitudes that can be achieved are adequate and whether the accretion/no-explosion phase lasts long enough to allow the core modes to excite to significance. Given these desiderata, Burrows et al. (2007) witness the explosion of all the progenitors they studied. Whether the acoustic mechanism, or a hybrid neutrino/acoustic mechanism, is relevant to supernova explosions remains to be seen, but it has several intriguing features, including the production of environments in which the r-process can occur and in which pulsar kicks are naturally imparted.

A third general class of explosion mechanisms, magnetohydrodynamic (MHD), has a long history (LeBlanc \& Wilson 1970; Bisnovatyi-Kogan et al. 1976; Meier et al. 1976; Symbalisty 1984). With the observation of collimated jets in the context of gamma-ray bursts (GRBs) and hypernovae (Nomoto et al. 2005; Mazzali et al. 2006; Woosley \& Bloom $2006)$, the association between GRBs and a small subset $(<0.5 \%)$ of supernovae, and the detection of signatures of asphericity in the generic supernova (Wang et al. 2002,2003), the MHD mechanism is experiencing a comeback (Ardeljan et al. 2000ab,2005; Wheeler et al. 2000; Wheeler, Meier, \& Wilson 2002; Akiyama et al. 2003; Yamada

\footnotetext{
${ }^{1}$ Department of Astronomy and Steward Observatory, The University of Arizona, Tucson, AZ 85721; burrows@as.arizona.edu,luc@as.arizona.edu,cott@as.arizona.edu,jmurphy@as.arizona.edu

${ }^{2}$ Racah Institute of Physics, The Hebrew University, Jerusalem, Israel; livne@phys.huji.ac.il
} 
\& Sawai 2004; Kotake et al. 2004; Mizuno et al. 2004; Takiwaki et al. 2004; Akiyama \& Wheeler 2005; Ohnishi, Kotake, \& Yamada 2005; Sawai, Kotake, \& Yamada 2005; Thompson, Quataert, \& Burrows 2005 (TQB); Proga 2005 ; Wilson, Mathews, \& Dalhed 2005; Moiseenko et al. 2006; Matt, Frank, \& Blackman 2006; Masada, Sano, \& Shibata 2007; Shibata et al. 2006). In addition, the possible role of magnetic winds in powering secondary explosions and in spinning down newly-born neutron stars or magnetars is being actively explored (Thompson, Chang, \& Quataert 2004; Bucciantini et al. 2006; Metzger, Thompson, \& Quataert 2006). While modern models of whatever stripe of supernova explosion are very asymmetrical, MHD models are fundamentally so.

However, all credible MHD models require rapid rotation, which the current crop of solar-metallicity supernova progenitor models do not have (Hirschi, Meynet, \& Maeder 2004,2005; Heger et al. 2000; Heger, Woosley, \& Spruit 2005). Pre-collapse spin periods of a few seconds may be needed, but Heger, Woosley, \& Spruit (2005), for example, derive spin rates at collapse ten times slower for the cores of solar-metallicity stars. The rotational kinetic energies at bounce are consequently one hundred times smaller than required and are totally inadequate to power a supernova explosion. However, are these progenitor calculations correct? Could the progenitor cores of massive stars before collapse actually be rotating fast? More conservatively, is there a subset of supernova progenitors that are spinning fast enough to make MHD processes relevant on explosion timescales? Importantly, the low-metallicity models of Woosley \& Heger (2006), some of which they suggest are GRB progenitors, have much faster spinning cores at collapse.

In this paper, we explore the physics and phenomenology of MHD-driven explosions when and if suitably rapid initial rotation rates obtain and leave open the question of the progenitor systems in which they might. Unlike all past investigations, we incorporate 2D multi-group neutrino radiation transport, a realistic equation of state, and realistic progenitor density, temperature, and composition structures. Our current supernova code, VULCAN/2D, has been upgraded to include MHD in 2.5D (Livne et al. 2007). We start at the onset of core collapse and follow the evolution from collapse, through bounce, to explosion, and all the way to many hundreds of milliseconds after explosion. We observe and document bipolar jet explosions due to magnetic stresses and buoyancy in magnetic towers (Uzdensky \& MacFadyen 2006ab), accretion and magnetic collimation of the jets, sausage/neck instabilities (Lampe 1991), and the growth to quasi-steady state of the MHD engine power. The jets are powered and maintained by the kinetic energy in differential rotation, which for these calculations is often significantly more than one Bethe. For the strongest jets, we observe core spindown by magnetic torques.

Akiyama et al. (2003) introduced into supernova theory the possibility that whatever the initial seed magnetic fields, the magneto-rotational instability (MRI; Balbus \& Hawley 1991; Pessah \& Psaltis 2005; Pessah, Chan \& Psaltis $2006)$, driven by the negative gradients in the angular velocity $(\Omega)$ after bounce that are unavoidable in the context of core collapse (Ott et al. 2006), could foster the growth to saturation values of poloidal $\left(B_{P}\right)$ and toroidal $\left(B_{\phi}\right)$ fields that are dynamically important. The recent calculations of Obergaulinger, Aloy, \& Müller (2006), Obergaulinger et al. (2006), Etienne, Liu, \& Shapiro (2006), and Shibata et al. (2006) make a good case that this is so. Hence, if the shear in $\Omega$ in the protoneutron star (PNS; Burrows \& Lattimer 1986) between 10 and 100 kilometers is large, we conclude that strong, if transient, magnetic fields with a dynamical role are inevitable in core collapse. In all situations with rapid rotation, the growth of B-fields due to compression, winding, and the MRI (and, perhaps, due to classical dynamo action) will play an essential role in the explosion mechanism. This paper is a preliminary exploration of this scenario and its consequences.

However, our simulations have a number of limitations. The calculations are Newtonian, are done in 2D (axisymmetric, plus rotation), and the transport is flux-limited (not multi-angle). Importantly, Etienne, Liu, \& Shapiro (2006) and Shibata et al. (2006) have shown that 2D spatial grids with thousands-squared of points are required to properly resolve the MRI in core-collapse simulations. Moreover, Obergaulinger, Aloy, \& Müller (2006) and Obergaulinger et al. (2006) have documented the dependence of the MRI growth rates on the initial, unknown, magnetic fields. Since we can't yet afford both high spatial resolution and multi-group neutrino transport, we have opted for the latter, since no calculation performed to date has had this central feature. Given our choice, we are still able to perform evolutions for not only tens of milliseconds, but for $\sim 1$ second and to witness the full panoply of dynamical phases.

In 92 , we discuss the overall physical context, motivate our approach to the simulations portrayed, and review the simple physics of flux compression and winding, as well as the basics of the MRI. We also describe many of the relevant timescales of the problem, all of which we argue are short compared with the time available in the explosion phase. This motivates the idea that a quasi-steady state engine is established and maintained. Furthermore, we present qualitative discussions of the anticipated dependence of the jet power on spin rate and of the free energy from differential motion available to power the MHD engine. The latter is continuously fed by equatorial accretion during the early phases of bipolar explosion. In \$3, we present our computational setup and the parameters of the simulations we describe. In $\$ 4$. we go on to describe and detail our simulation results and the phenomenology and morphology of the resultant blasts. We discuss the magnetic tower phenomenon, the poloidal and toroidal field distributions, the emergence of the jet and its collimation, the weak sausage instability, and the power density distributions. In \$5 we discuss the magnitude and evolution of the spin rates and the free energy of differential rotation. We also explore the initial spin-rate dependence of the latter. Section 6 presents estimates of the derived jet powers and explosion energies and we conclude in $\$ 7$ with both a summary of our central results and speculations on their wider astrophysical import.

\section{THE OVERALL PHYSICAL CONTEXT}

A progenitor core will have initial rotational and magnetic field profiles. Magnetic torques and wind mass loss during the pre-collapse evolution of the star will help determine both these distributions. Yet despite recent detailed efforts to model supernova progenitors (e.g., Heger, Woosley, \& Spruit 2005; Hirschi, Meynet, \& Maeder 2004,2005), we still have 
no reliable constraints on the spin or magnetic structures of the white-dwarf-like core that collapses when achieving the Chandrasekhar mass. For instance, the predictions for the spin period, $P_{0}$, of the Chandrasekhar core vary from $\sim 1.5$ seconds to $\sim 50$ seconds. This involves a range in the possible initial rotational kinetic energies of $\sim 10^{3}$. There is a similarly wide range of predictions for the magnitudes of the pre-collapse toroidal and poloidal fields and there are no predictions for 3D structures that are consistent with Maxwell's equations.

However, if the initial spin rate and angular momentum are large, and the MRI (or dynamo action in 3D) occurs in the protoneutron star after bounce, then the resulting magnetic energy will be expected to exponentiate to saturation values that are in rough equipartition with the rotational free energy in the post-bounce differential motion. The growth of B-field energy due to the MRI instability feeds off the free energy available in differential rotation, which is the difference between the rotational kinetic energy and the kinetic energy for solid-body rotation at the same total angular momentum. For example, Shibata et al. (2006) find that the MRI saturates in the core-collapse context at magnetic energies that are very roughly $10 \%$ of the rotational energy. The degree to which the free energy is close to the total rotational energy itself depends upon the magnitude and distribution of the angular-velocity and density gradients. In particular, if the post-bounce core, which may contain a lot of the rotational energy, is in near solid-body rotation, and most of the shear is in an outer fraction of the mass (albeit with larger moment arms), then a smaller fraction of the total rotational energy will be available to pump up the magnetic energy by the MRI. This fraction could be only $10 \%$, but might be $10 \%-50 \%$. For instance, the work of Ott et al. (2006) suggests that progenitor cores with a fast $P_{0}$ of 2.34 seconds, can have rotational free energies at $\sim 200$ milliseconds (ms) after bounce that range from $\sim 2$ to $\sim 12$ Bethes, depending upon progenitor mass and model. Progenitor cores with slower $P_{0} \mathrm{~s}$ of $\sim 10$ seconds can have free energies (at the same epoch) that range from $\sim 0.1$ to $\sim 0.2$ Bethes, while those with a slow $P_{0}$ of $\sim 50$ seconds can have rotational free energies of only $\sim 0.03$ Bethes. Hence, depending upon the initial spin profile, the free energy available in differential rotation to be tapped to generate strong magnetic fields and MHD jets varies from insignificance to supernova and hypernova magnitudes.

In this paper, we explore the consequences for supernova theory of rapid initial rotation that should lead to strong magnetic fields and MHD-jet-driven explosions. We envision initial $P_{0}$ s less than $\sim 4$ seconds, but focus for specificity on a $P_{0}$ of 2 seconds. Such rapid spin rates may or may not obtain, or may obtain rarely and/or only in the hypernova context, but this is not our concern here. Since with our modest number of grid points (\$3) we can not fully resolve the MRI (see \$1), we hypothesize (and calculate) that the MRI growth rates are sufficiently fast that within 100-200 milliseconds after bounce the resulting MHD jet structure will stabilize on longer-term secular evolution times, such as the accretion and neutrino cooling times (0.5-10 seconds), and will lose track of the initial B-field configuration. In this way, to a reasonable approximation, the structure, hydrodynamic flow, rotational profiles, and jet power will settle into a long-term evolution that depends most centrally only on the initial spin and thermodynamic profiles, and not on the initial B-fields (at least, not very much). Such should be a consequence of quick exponential growth to saturation. This ansatz motivates our choices of the initial poloidal and toroidal fields (\$3); these are set to lead by flux compression and rotational field winding to final B-fields whose magnitudes roughly approximate those expected due to the MRI at and around saturation. For a $P_{0}$ of 2 seconds, the resulting toroidal fields between $\sim 10$ kilometers $(\mathrm{km})$ and $\sim 100 \mathrm{~km}$ turn out to be very roughly $10^{15 \pm 0.5}$ Gauss $(\mathrm{G})$. In $\$ 2.3$, we provide a very approximate analytic connection between period and saturation B-field.

Since flux freezing, field winding on infall, field winding after infall, and amplification due to the magneto-rotational instability (MRI), of both poloidal and toroidal field components, are of interest, we next provide short discussions of each in turn, with a focus on the relevant timescales and approximate magnitudes. None of this is original, but we include it for general context. Note that the various timescales all scale with the rotational period. Hence, in the case of rapid rotation, all the relevant processes are accelerated and should lead to a jet-driven explosion that achieves a quasi-steady state on a "short" timescale. After the asides in $\$ 2.192 .2$ and 92.3 on order-of-magnitude estimates, we describe in $\$ 3$ our computational and initial model setup, and then transition in $\$ 4$ to a detailed discussion of our simulation results.

\subsection{Field Amplification and Timescales}

Rotational winding converts/stretches poloidal field into toroidal field. The basic equation:

$$
\frac{\partial B_{\phi}}{\partial t} \sim B_{P}\left(\frac{\partial \Omega}{\partial \ln r}\right)
$$

leads to

$$
\Delta B_{\phi} \sim B_{P} \Omega t \sim B_{P}\left(\frac{2 \pi t}{P}\right) .
$$

In eq. (2), $t$ is the time, $P$ is the local spin period, and $\Omega$ is the corresponding angular frequency. Eq. (2) suggests that each turn leads to a boost in $B_{\phi}$ near $2 \pi$ and that growth is linear in $t$.

On infall, winding alone yields

$$
\frac{\Delta B_{\phi}}{B_{P}} \sim \Omega_{0} \int\left(\frac{r_{0}}{r}\right)^{2} d t \sim 3 \Omega_{0} t_{0}\left(\frac{r_{0}}{r}\right)^{1 / 2} \sim 60 \pi\left(\frac{t_{0}}{P_{0}}\right)
$$


where we have used $r^{3 / 2} \sim r_{0}^{3 / 2}\left(t_{0}-t\right) / t_{0}$ and angular momentum conservation: $\Omega=\Omega_{0}\left(\frac{r_{0}}{r}\right)^{2}$. $t_{0}$ is the time to bounce ("collapse time"), $r_{0}$ is the initial radius of the collapsing shell, and $P_{0}$ is the initial rotation period. With $t_{0}=0.2$ seconds, $P_{0}=20$ seconds, $r_{0} \sim 2000 \mathrm{~km}$, and $r \sim 20 \mathrm{~km}, \frac{\Delta B_{\phi}}{B_{P}} \sim 2$, with a reasonable range between 1 and 10. However, if $P_{0}=2$ seconds, this ratio is ten times larger.

Core collapse leads to matter compression. Magnetic flux conservation would then amplify both the toroidal and the poloidal fields. The magnitude of the compression is $\sim\left(\frac{\rho}{\rho_{0}}\right)^{2 / 3}$. With $\rho \sim 3 \times 10^{14} \mathrm{~g}^{\mathrm{cm}^{-3}}$ and $\rho_{0} \sim 7 \times 10^{9} \mathrm{~g}$ $\mathrm{cm}^{-3},\left(\frac{\rho}{\rho_{0}}\right)^{2 / 3} \sim 10^{3}$. If we combine the prescription for winding on infall with flux compression, assuming that the collapse is homologous $\left(\frac{\rho}{\rho_{0}} \sim\left(\frac{r_{0}}{r}\right)^{3}\right)$, we find

$$
\frac{\Delta B_{\phi}}{B_{P}} \sim \frac{3}{5} \Omega_{0} t_{0}\left(\frac{r_{0}}{r}\right)^{5 / 2} .
$$

This expression is within a factor of two of the product of flux compression and winding (eq. 3) on infall taken separately. Given the parameters above, flux compression yields a factor of $\sim 10^{3}$ and winding a factor of a few (again, for an initial period of $\sim 10-20$ seconds). Note that the winding amplifies only the toroidal component, while flux compression amplifies both $B_{\phi}$ and $B_{P}$. Flux compression alone takes a toroidal field from $\sim 10^{10} \mathrm{G}$ to $\sim 10^{13} \mathrm{G}$ and a poloidal field from $\sim 10^{7} \mathrm{G}\left(B_{P}^{0}\right)$ to $\sim 10^{10} \mathrm{G}\left(B_{P}\right)$. Using eq. (2), and starting with a $B_{P}$ of $10^{10} \mathrm{G}$, we can derive the time to achieve a given change in the toroidal field due solely to flux winding:

$$
t_{\text {wind }} \sim 32 \mathrm{~ms}\left(\frac{P}{2 \mathrm{~ms}}\right)\left(\frac{\Delta B_{\phi}}{10^{12} \mathrm{G}}\right)\left(\frac{10^{10} \mathrm{G}}{B_{P}}\right)
$$

If the initial fields are not very large, winding by itself, and not aided by the MRI or dynamo action in 3D, can require a long time to achieve dynamically interesting field strengths $\left(\sim 10^{15} \mathrm{G}\right)$. It is, nevertheless, a very important process in rotational supernovae, particularly if the MRI or a dynamo can deliver after bounce and on exponential timescales high values of the poloidal field.

\subsection{Amplification by Magneto-Rotational Instability}

In local linear mode analysis, one can derive a characteristic dispersion relation that depends on the rotational shear $\left(\frac{\partial \Omega}{\partial \ln r}\right)$, the magnitude of the B-field, and the Brunt-Väisälä frequency (Balbus \& Hawley 1991; Pessah \& Psaltis 2005; Akiyama et al. 2003). Ignoring the Brunt-Väisälä frequency, the maximum rate of MRI modal amplification occurs for a given wavenumber, $k_{m r i} \sim \Omega / v_{A}$, where $v_{A}$ is the Alfvén speed, and has an associated growth time $\left(\tau_{m r i}\right)$ of

$$
\tau_{m r i} \sim 4 \pi\left(\frac{\partial \ln r}{\partial \Omega}\right) \sim 2 P,
$$

where $P$ is the spin period at and after bounce. Note that $\tau_{m r i}$ is proportional to $P$, and that if $P \sim 2 \mathrm{~ms}, \tau_{m r i}$ is also on millisecond timescales.

The Alfvén speed is given by

$$
v_{A}=\frac{B}{\sqrt{4 \pi \rho}} \sim 10^{9} \mathrm{~cm} \mathrm{~s}^{-1} \frac{B_{15}}{\rho_{11}^{1 / 2}},
$$

where $\rho_{11}$ is the mass density in units of $10^{11} \mathrm{~g} \mathrm{~cm}^{-3}$ and $B_{15}$ is the field strength in units of $10^{15} \mathrm{G}$. With eq. (77), we see that the physical scale of the mode with the largest MRI growth rate is approximately:

$$
\lambda_{m r i}^{\max } \sim \frac{2 \pi v_{A}}{\Omega} \sim v_{A} P \sim 10^{4} \mathrm{~cm} P_{10} \frac{B_{12}}{\rho_{11}^{1 / 2}},
$$

where $P_{10}$ is the rotation period in units of 10 milliseconds. Eq. (8) shows that for "small" initial seed fields, $\lambda_{m r i}^{m a x}$ can, in a computational sense, be quite small.

Etienne et al. (2006) note that the characteristic time for tapping differential rotation, transferring angular momentum by magnetic torques, and saturating the MRI is the Alfvén crossing time:

$$
t_{A}=\frac{R}{v_{A}} \sim 10 \mathrm{~ms} \frac{R_{7}}{B_{15}} \rho_{11}^{1 / 2}
$$

where $R_{7}$ is the radius in units of $10^{7} \mathrm{~cm}$. Hence, when high fields are achieved, $t_{A}$ is quite short in evolutionary terms.

Finally, using eq. (6), exponential growth of the MRI yields the time, $t_{m r i}$, it takes to achieve a field of $B_{f}$, starting from a field of $B_{i}$, of

$$
t_{m r i} \sim 20 \mathrm{~ms}\left(P_{10}\right) \ln \frac{B_{f}}{B_{i}} \sim\left[92+20 \ln \left(\frac{B_{f}}{10^{15} \mathrm{G}}\right)\left(\frac{10^{13} \mathrm{G}}{B_{i}}\right)\right]\left(P_{10}\right) \mathrm{ms} .
$$

Eq. (10) suggests that rapid spin rates can deliver fields of $\sim 10^{15} \mathrm{G}$ within $100-200$ milliseconds, with a weakish dependence on $B_{i}$. 
The approximate formulae from eq. (6) to eq. (10) suggest that all the relevant timescales scale with $P$, that they can be quite short, and that if the MRI obtains it can generate dynamically interesting fields within 100-200 milliseconds. Furthermore, eq. (5) demonstrates that subsequent rotational winding can act to maintain high fields as long as the rotational energy of the core is maintained.

We end this subsection by providing in Fig. 1 representative colormaps of the peak linear MRI growth timescale, $\tau_{m r i}$ (left-hand side), and the associated wavelength, $\lambda_{m r i}^{\max }$ (right-hand side). These numbers were obtained using the output of our 2D radiation/hydro simulation, M15B11UP2A1H (see §3), 10 milliseconds after bounce and the formalism of Pessah \& Psaltis (2005). Figure 1 shows that in the inner $50 \mathrm{~km}, \tau_{m r i}$ in our calculations is only 10-30 $\mathrm{ms}$, but that $\lambda_{m r i}^{\max }$ is 0.1 to $0.5 \mathrm{~km}$. In these regions, our spatial resolution is on the order of $0.5 \mathrm{~km}$, and since Etienne et al. (2006) have shown that $\sim 10$ resolution elements over $\lambda_{m r i}^{\max }$ are needed to fully resolve the growth of the MRI, our calculations fall short in this regard. Were we to attempt the resolution required, we would need to increase the total number of zones employed in our calculations by more than an order of magnitude, rendering our 2D, multi-group radiation hydrodynamic calculations extremely expensive and time-consuming. Worse still, model M15B11UP2A1H incorporates a large initial poloidal field, which yields an overlarge $\lambda_{m r i}^{\max }$. Had we used an even smaller, more realistic, initial $B_{\phi}, \lambda_{m r i}^{\max }$ would have been correspondingly smaller (eq. 8), putting its resolution even further out of reach for our simulations with multi-group radiation that are evolved for many hundreds of milliseconds past bounce. Since each simulation presented in this paper already consumes about a month on modern supercomputers, one can see why we have opted for our current strategy. That strategy involves using high initial poloidal fields $\left(B_{P}\right)$ that nevertheless result in toroidal and poloidal fields after bounce that are of similar magnitude to what could be expected given the saturation of a fully functioning MRI. We did calculations for a spectrum of initial conditions, but highlight only models M15B11UP2A1H and M15B11DP2A1H which very approximately result in the requisite fields. We now turn to back-of-the-envelope estimates of those saturation fields as a function of $\Omega$.

\subsection{Expected Systematics with $\Omega$ of Saturation Fields and Jet Power}

At saturation, the energy in the magnetic field in the post-bounce region experiencing significant rotational shear should scale with the kinetic energy in that differential motion (the "free energy" of rotation). The inner core, with a radius of $\sim 10 \mathrm{~km}$, is generally in solid-body rotation, and, hence, is not a factor in generating new field by the MRI (Akiyama et al. 2003). The region of interest is between $\sim 10 \mathrm{~km}$ and $\sim 100 \mathrm{~km}$. Following the arguments of Shibata et al. (2006) and Hawley, Gammie, \& Balbus (1996), to determine saturation fields we can set either a fraction $(\varepsilon \sim 0.1)$ of the energy density in shear motion equal to the magnetic energy density, or set the square of the Alfvén speed equal to that same fraction of $(r \Omega)^{2}$, where $r$ is the cylindrical radius. Approximately, this yields:

$$
B \sim \sqrt{4 \pi \varepsilon \rho r^{2} \Omega^{2}} \sim 10^{15} \mathrm{G}\left(\frac{\varepsilon}{0.1}\right)^{1 / 2}\left(\rho_{11} r_{30}^{2}\right)^{1 / 2}\left(\frac{\Omega}{10^{3} \mathrm{rad} \mathrm{s}^{-1}}\right),
$$

where $r_{30}$ is the radius in units of 30 kilometers. Of course, the fields generated will have spatial distributions, as do $\rho$ and $\Omega$, and $\Omega$ generally decreases with $r$. Eq. (11) merely hints at the scalings and magnitudes expected. Importantly, it shows that $B$ is expected to be linear with $\Omega$ and that $\Omega$ s near $\sim 10^{3}$ translate into fields near $10^{15} \mathrm{G}$. Ott et al. (2006) have shown that the ratio of initial spin period to spin period at and after bounce is roughly $\sim 10^{3}$. So an $\Omega$ of $10^{3} \mathrm{rad} \mathrm{s}^{-1}$ after bounce implies a $P_{0}$ near $\sim 5$ seconds. Therefore, for such initial progenitor periods, saturation fields of around $\sim 10^{15} \mathrm{G}$ at radii of tens of kilometers are reasonable.

The spindown torque due to a magnetic jet should be proportional to $B_{\phi} \times B_{P}$. Since field winding will ensure that $B_{\phi}>B_{P}$, the torque is proportional to $\frac{B_{P}}{B_{\phi}}$ times the total magnetic energy in the MRI/shear region. As eq. (11) implies, the latter is proportional to $\Omega^{2}$ and since the jet power $(\dot{E})$ is approximately equal to $\Omega$ times the torque, $\dot{E}$ is proportional to $\Omega^{3}$ (Shibata et al. 2006). This is a very useful result. More quantitatively,

$$
\dot{E} \sim \varepsilon\left(\frac{B_{P}}{B_{\phi}}\right) F_{r o t} \Omega \sim 10^{52} \operatorname{ergs~s}^{-1}\left(\frac{\Omega}{10^{3} \mathrm{rad} \mathrm{s}^{-1}}\right)^{3},
$$

where $F_{r o t}$ is the free energy in differential rotation and we have set $\frac{B_{P}}{B_{\phi}}$ equal to 0.1 (Shibata et al. 2006; Etienne et al. 2006). Since the outer post-shock region is rotating more slowly than the inner region, if $\Omega$ equals $10^{3} \mathrm{rad} \mathrm{s}^{-1}$ at $\sim 10-30$ kilometers, using $10^{3}$ in eq. (12) is likely to be a slight overestimate. Nevertheless, jet powers of $10^{51-52} \mathrm{ergs}$ $\mathrm{s}^{-1}$ for $\Omega \mathrm{s}$ near $10^{3} \mathrm{rad} \mathrm{s}^{-1}$ are sensible. Such powers will pump supernova-like energies into the ejecta within 0.1 to 1.0 seconds. This conclusion is the essence of our study and our simulations bear it out.

It should be noted that Thompson, Quataert, \& Burrows (2005), using the analogy of $\alpha$-disk accretion, have explored the local heating rate due to field dissipation in a region experiencing the MRI and derive a $\Omega^{3}$ dependence for the associated heating power. In fact, eq. (12), representing large-scale power and the TQB formula, representing the associated small-scale dissipational heating, yield numbers that are within an order of magnitude of one another. So, our modest-resolution simulations may be underestimating the driving power of a saturated MRI.

Finally, it is instructive to compare eq. (12) with the corresponding formula for the magnetic dipole radiation of a pulsar into a vacuum:

$$
\dot{E}_{p u l} \sim\left(\frac{B_{\perp}^{2} R^{3}}{6}\right)\left(\frac{R \Omega}{c}\right)^{3} \Omega
$$


where $B_{\perp}$ is the surface field perpendicular to the rotation axis. The first term in eq. (13) is near the total energy in the magnetic field. That, multiplied by $\frac{B_{P}}{B_{\phi}}$ and $\Omega$, is an approximate form for eq. (12), without the assumption that $B \propto \Omega$. However, eq. (13) contains the term $\left(\frac{R \Omega}{c}\right)^{3}$, which is the cube of the ratio of the radius of the acceleration region to the light-cylinder radius and is a small number $\left(\sim 10^{-3}\right)$ for $\Omega$ s near $10^{3} \mathrm{rad} \mathrm{s}^{-1}$. So, the power of the MHD-driven jets we investigate in this paper, $\dot{E}$, generally trumps, at least in the first seconds after bounce, $\dot{E}_{p u l}$. Note that the characteristic dipole spindown time (time to "halve" the initial period) is only days for a magnetar with $B_{\perp}=10^{15} \mathrm{G}$ and an initial spin period of $10 \mathrm{~ms}$.

Eq. (12) indicates that $\dot{E} \propto F_{\text {rot }}$. Since $F_{\text {rot }}$ might be considered the energy reservoir being tapped for the MHD jet, their ratio is a measure of the characteristic time $\left(t_{j e t}\right)$ during which the jet operates. Remarkably, $t_{j e t}$ is approximately equal to $\frac{P}{0.1 \varepsilon(2 \pi)}$, demonstrating that yet another time scales with period. In fact, $t_{j e t}$ is roughly $10-20 \times P$ and, if $P=4 \mathrm{~ms}, t_{j e t} \sim 60 \mathrm{~ms}$. This is after saturation is achieved, which might take 100-200 ms, but is still rather short. However, as we will see, $F_{\text {rot }}$ is constantly replenished by equatorial accretion during polar explosion. In summary, 2.1 2.2. and $\$ 2.3$ collectively demonstrate that rapid rotation leads to magnetic field strengths, energies, and characteristic timescales that are very relevant in the context of collapse dynamics and the associated supernova.

\section{NUMERICAL TECHNIQUE AND COMPUTATIONAL SETUP}

We perform the calculations presented in this paper with the code VULCAN/2D (Livne et al. 2004; Walder et al. 2005; Ott et al. 2006; Dessart et al. 2006ab; Burrows et al. 2006,2007), newly enhanced with an ideal MHD capability (Livne et al. 2007). VULCAN/2D solves the multi-energy-group, 2D radiation-magnetohydrodynamic (RMHD) equations of fluid motion with rotation. The toroidal component of the magnetic field $\left(B_{\phi}\right)$ is axisymmetric. Otherwise, the three B-field components in cylindrical coordinates $\left(B_{\phi}, B_{z}\right.$, and $\left.B_{r}\right)$ are evolved using the corresponding Maxwell's equations in the MHD approximation with infinite conductivity. The magnetic stresses are fully incorporated into the matter momentum equations. The divergence-free condition of the B-field is maintained to machine precision.

For this study, we use 16 energy groups for each of three neutrino species $\left(\nu_{e}, \bar{\nu}_{e}\right.$, and " $\nu_{\mu}$ ") and a 2D flux-limiter (Burrows et al. 2006,2007). Hence, the calculations are not multi-angle, though VULCAN/2D has this capability (Livne et al. 2004). Redistribution due to neutrino-electron scattering, a subdominant process with respect to the charged-current URCA processes involving free nucleons, is ignored and velocity-dependent terms in the transport equations have been dropped. The latter approximation can underestimate the degree of neutrino heating in the gain region by $\sim 10 \%$ (Hubeny \& Burrows 2007).

We use the hybrid grid that we have employed in previous VULCAN/2D multi-D radiation-hydrodynamics simulations (Ott et al. 2006; Dessart et al. 2006ab; Burrows et al. 2006,2007). The grid is spherical-polar outside of a transition radius, here set equal to $25 \mathrm{~km}$, and switches smoothly to a pseudo-Cartesian grid inside, extending right down to the center. Using 221 grid points, we adopt a constant radial increment in the log between the transition radius at $25 \mathrm{~km}$ and the maximum radius, $5000 \mathrm{~km}$, of the computational domain. We use 71 equally-spaced angular zones over a $90^{\circ}$ angular domain from the rotation axis to the equator (but see M15B10DP2A1F ${ }^{3}$ below) to ensure that cells have an aspect ratio of about unity throughout the spherical-polar region ${ }^{4}$. The total number of zones is 17909. The highest resolution is achieved at the center. Because of the Cartesian gridding there, resolution is limited to $\sim 350$ meters to guarantee reasonably "large" Courant times of no less than about a microsecond. The lowest resolution is at the outer radius of $5000 \mathrm{~km}$ and is $\sim 110 \mathrm{~km}$.

The progenitor employed for the simulations reported in the present study is the rotating $15-\mathrm{M}_{\odot}$ model (m15b6) of Heger, Woosley, \& Spruit (2005, HWS). The cores of the HWS suite of models experience significant magnetic spindown during stellar evolution to collapse and generally leave very slowly rotating cores, with central periods from $\sim 30$ to $\sim 50$ seconds. The HWS $15-\mathrm{M}_{\odot}$ model has an interior period near $\sim 30$ seconds. Although this family of progenitor models was evolved by HWS in 1D with a well-motivated prescription for rotation, magnetic fields, and their evolution, we use instead our own analytical description for the initial angular-velocity and magnetic field profiles and spin this initial model up significantly in order to explore the effects of rapid rotation. The initial rotation law is given by $\Omega(r)=\frac{\Omega_{0}}{1+\left(r / A_{0}\right)^{2}}$, where $r$ is the cylindrical radius, for which we choose $\Omega_{0}=\pi \mathrm{s}^{-1}$ (initial inner core period of 2 seconds) and $A_{0}=1000 \mathrm{~km}$. We adopt two initial divergence-free field configurations: 1) the poloidal and toroidal fields are uniform throughout the computational domain (this is our "uniform" [U] configuration) and 2) the poloidal and toroidal fields are uniform in the cylindrical $z$ direction within a sphere of radius $A_{0}$ and dipolar (falling off as $1 / r^{3}$ ) outside. This is our " $\mathrm{D}$ " configuration. In the latter setup, the toroidal component is taken to have the same radial dependence as the magnitude of the poloidal component.

We reiterate that our simulations are axisymmetric and, thus, that no variable has a $\phi$-angle dependence. In the full study (a subset of which is reported here), the magnitude of the initial poloidal and toroidal fields was varied. We focus here on models with initial $B_{z}=10^{11}$ Gauss $(\mathrm{G})$ in the center, which we refer to as models M15B11DP2A1H and M15B11UP2A1H and the initial toroidal magnetic field $\left(B_{\phi}\right)$ is chosen to be 3 orders of magnitude smaller than the polodial component. The model naming convention we follow is $\mathrm{M} 15 \mathrm{BA}^{\prime} \mathrm{A}^{\prime} \mathrm{B}^{\prime} \mathrm{PCAD}^{\prime} \mathrm{H}$, where $\mathrm{A}^{\prime} \mathrm{A}^{\prime}$ is the $\log$ of the initial poloidal field in Gauss (e.g., 10, 11, or 12), B' is the B-field configuration given above (either "U" or

\footnotetext{
${ }^{3} \mathrm{~F}$ means that the calculations were done on the full $180^{\circ}$ semicircle.

${ }^{4}$ Note that limiting the computational domain to $90^{\circ}$ suppresses the $\ell=1$ SASI (standing accretion shock instability) and core g-modes (Burrows et al. 2006,2007).
} 
"D"), C is the initial central spin period $\left(=2 \pi / \Omega_{0}\right)$ in seconds (here either 2 or 4 seconds), $\mathrm{D}^{\prime}$ is $A_{0} / 1000 \mathrm{~km}$, and $\mathrm{H}$ means that the calculations were done on a $90^{\circ}$ quadrant. We have performed many simulations with a variety of parameters, but focus here on $\mathrm{A}^{\prime} \mathrm{A}^{\prime}=11$. Most previous purely MHD simulations (see \$1) focussed on models with initial fields at the high end, in fact near $10^{12-13}$ Gauss, in order to obtain some dynamical effect on the short timescales (tens of milliseconds) they could address. Table 1 lists the simulations that have informed our investigation and conclusions, and includes general information on each run. Note that model M15B0DP2A1H is the non-magnetic model we include to gauge the effects of rapid rotation alone and employs a slightly altered naming convention. Model M15B11DP4A1H explores the dependence on $P_{0}$ and model M15B12DP2A1H explores the effects of very large fields that might perhaps be too large for an assumed $P_{0}$ of 2 seconds, but nevertheless demonstrates several interesting, and dramatic, features. Model M15B10DP2A1F is calculated over the full $180^{\circ}$ angular realm, thereby liberating the $\ell=1$ mode of the standing accretion shock instability (SASI; Blondin, Mezzacappa, \& DeMarino 2003; Foglizzo, Scheck, \& Janka 2006; Buras et al. 2006ab; Burrows et al. 2007). With model M15B10DP2A1F, we can determine the influence of rotation and magnetic fields on the SASI. Note that all these models explode before the core g-modes would have had a chance to achieve significant amplitudes (Burrows et al. 2006,2007).

\section{SIMULATION RESULTS}

All the RMHD collapse models we have simulated go through the same general stages and manifest the same phenomena. Within $\sim 10-30 \mathrm{~ms}$ of bounce, the shock wave launched at bounce stalls near $100-150 \mathrm{~km}$ in the canonical fashion (Bethe \& Wilson 1985; Wilson 1985; Burrows et al. 2006,2007; Buras et al. 2006ab). Differential rotation in the cavity between the stalled shock and the inner PNS core inexorably winds the toroidal component of the B-field to higher and higher values (2.1). Eventually, the magnetic stresses due to the amplified $B_{\phi} \mathrm{s}$ achieve values in a quasi-cylindrical column along the poles that begin to compete with the gas pressures $\left(P_{\text {gas }}\right)$. In particular, magnetic stresses $\left(P_{\mathrm{mag}}\right)$ just behind the shock start to rival the local $P_{\text {gas }}$. When this happens, a magnetically-driven jet punches into the accreta along the poles, powering a bipolar explosion. Figure 2 provides six colormaps of the ratio, $P_{\text {mag }} / P_{\text {gas }}$, for model M15B11DP2A1H just before, during, and after the onset of explosion. Note that the explosion commences only when this ratio reaches a value near unity (dark blue) along the poles, at which time the jet emerges. The last five stills are separated from one another by only 10 milliseconds, indicating that when the pressure condition is achieved the explosion does not long delay. Figure 3 portrays the evolution of this ratio along the poles for three representative models over a wider time span. The importance of the $P_{\text {mag }} / P_{\text {gas }} \sim 1$ condition is manifest in all these as well, even though the epoch of explosion is very different for each model (see caption). For each line, a vertical drop traces the shock wave, which starts post-bounce life as an accretion shock, but then transitions into a propagating explosion when the $P_{\text {mag }} / P_{\text {gas }} \sim 1$ condition is reached.

The structure of the magnetic field in the resultant cavity is that of a tightly coiled spring, and the explosion resembles a "magnetic tower" (Uzdensky \& MacFadyen 2006ab). Radial gradients in the hoop stresses along both rotation axes (top and bottom) are responsible for the jet thrust. At the inauguration of the jet, the magnetic stresses in the equatorial sector are not yet competitive with the local gas pressures. Note that all that is required for a jet to emerge to reenergize the shock and to overcome the local ram pressure of the accreting matter is the focussed application of magnetic pressure, such as occurs first along the rotation axis. Hence, even a "small" MHD power (but over a small solid angle) can "bore a hole." The jet is confined predominantly by the accreting matter through which it drills, and by the magnetic hoop stresses. Since the jet is thereby inhibited from spreading laterally, it is not centrifugally flung out to large angles and, hence, does not resemble a Blandford-Payne jet (Blandford \& Payne 1982). For all our runs, which extend up to $\sim 1$ second after bounce (Table 1) and achieve jet penetrations of $\sim 5000 \mathrm{~km}$, the jet remains tightly collimated and does not diverge.

The jet-driven explosions we obtain are not energized by the impulsive deposition of energy, but by the continuous injection of power due to magnetic stresses in the inner core that are continuously replenished by winding of poloidal field into "new" toroidal field. The ultimate source of energy and power is the gravitational energy of infall, converted into differential rotational motion of the shocked outer core, and then transformed into magnetic energy. The postbounce time needed to launch the jet varies in our suite of models from $\sim 80 \mathrm{~ms}(\mathrm{M} 15 \mathrm{~B} 12 \mathrm{DP} 2 \mathrm{~A} 1 \mathrm{H})$ to $\sim 550 \mathrm{~ms}$ $(\mathrm{M} 15 \mathrm{~B} 10 \mathrm{DP} 2 \mathrm{~A} 1 \mathrm{H})$, but in all cases is much shorter than the time it would take to inject into the explosion at the jet powers achieved all the free energy that resides in core shear motion. In other words, the jets achieve a quasisteady state of roughly constant power that changes only on secular evolution timescales. An "engine" is established. Furthermore, jet explosion along the poles is simultaneous with continuing accretion along the equator. Such accretion brings in "fresh" rotational energy that maintains the store of kinetic energy being tapped by the magnetic jet. The free energy available to the MHD jet actually continues to grow after bounce. Hence, accretion does not inhibit explosion, but powers it. This is reminiscent of the situation with the acoustic mechanism (Burrows et al. 2006,2007), in which there is also simultaneous accretion and explosion. As in that case, the breaking of spherical symmetry into dual matter streams is the dynamical key.

Figure 4 depicts snapshots of the distribution of magnetic "streamlines" anchored in footpoints at arbitrary positions in the inner regions. The left panel shows model M15B11UP2A1H at $264.5 \mathrm{~ms}$ after bounce and the right panel shows model M15B10DP2A1H at $855.5 \mathrm{~ms}$ after bounce. The lines are colored in entropy, with the yellow/red lines tracing the shocked regions and the light blue lines tracing the unshocked regions. The severe twisting of the field lines due to rotational winding behind the shock is clearly seen, as is the magnetic tower morphology ${ }^{5}$. Moreover, the

5 The vertical coiled spring structure depicted in Figure 4 and in Figure 5 below, makes clear why this field distribution is referred to 
"auger" or "punch" shape of the confined jet is manifest. Model M15B10DP2A1H has a more tightly wound field structure because its lower initial poloidal component (M15 "B10" DP2A1H) necessitated more rotations to achieve the requisite magnetic pressures and because the resulting explosion power and ejection speeds were lower. Therefore, the spring unwound more slowly. Nevertheless, models M15B11UP2A1H and M15B10DP2A1H are qualitatively, if not quantitatively, the same.

Figure 5 portrays streaklines of the trajectories of marker particles covering the last $200 \mathrm{~ms}$ of the M15B11UP2A1H (left) and M15B11DP2A1H (right) simulations. The lines are the paths followed by representative parcels of matter that are co-extensive with the magnetically-driven jet. Figure 5 clearly shows the lift-off of the cork-screwing Lagrangian parcels as rotation transitions into spiraling ejection, and then, at larger radii, into a directed jet. In addition, in model M15B11UP2A1H the radius of the shock in the equatorial regions is larger. This is because the equatorial magnetic pressures achieved there at a given time are larger than in model M15B11DP2A1H. This, in turn, is due to the fact that in model M15B11UP2A1H the uniform ("U") initial poloidal field results in larger accreted fields at later times than in model M15B11DP2A1H, for which the late-time accretion is of matter from the outer core where the initial field decays in the $\frac{1}{r^{3}}$ dipolar manner (\$3). In fact, for model M15B11UP2A1H the equatorial regions join the explosion at later times. This outcome is expected eventually for all models, but due to the different magnetic field structures and magnitudes for the models listed in Table 1 the times to equatorial explosion will vary greatly from model to model.

The particle trajectories implied by Fig. 5 and magnetic flux freezing indicate that the ejected material stretches toroidal field into poloidal field, in a reverse of what happens during rotational winding in the inner $\sim 20-150 \mathrm{~km}$. So, in the jet column at large radii the field has a significant poloidal component.

Figure 6 shows radial slices along the poles (solid lines) and along the equator (broken lines) of both the poloidal (red) and toroidal (black) fields for models M15B11DP2A1H (left) and M15B11UP2A1H (right) at $635 \mathrm{~ms}$ and 585 $\mathrm{ms}$, respectively, after bounce. Since there is no appreciable rotational shear interior to $\sim 10 \mathrm{~km}$, the magnetic fields there have little dynamical effect. It is the fields in the region between $\sim 10 \mathrm{~km}$ and $\sim 150 \mathrm{~km}$ that are of consequence, since it is here that the magnetic tower is launched and maintained. Figure 6 and $\$ 2.3$ indicate that the fields achieved in this region in these models are comparable to what is expected at saturation for a $P_{0}$ of 2 seconds $\left(\sim 10^{15} \mathrm{G}\right)$. This justifies our focus on these models when assuming $P_{0}=2$ seconds, despite the fact that we underresolve the MRI.

Figure 7 depicts colormaps of the poloidal (left) and toroidal (right) field distributions in model M15B11UP2A1H, $585 \mathrm{~ms}$ after bounce. In both panels, the lines are iso-poloidal field lines and the inner $200 \mathrm{~km}$ on a side is shown. The relative extents of the red and yellow regions demonstrate the dominance of the toroidal component in the inner zones at these late times well into the explosion, but the presence of a column of yellow/red (high-field) along the axis in the poloidal plot attests to the conversion due to stretching by ejected matter of toroidal into poloidal field (see also Fig. 51). Figure 7 also demonstrates the columnar structure of this inner region due both to equatorial accretion (and, hence, pinching) and rotation about the (vertical) axis. However, it should be made clear that the actual field distributions after saturation are likely to be different, and what they are in detail when the MRI is fully enabled remains to be determined.

Figure 8 compares maps of the the gas pressures $\left(P_{\text {gas }}\right.$, left $)$ with the magnetic pressures $\left(P_{\text {mag }}\right.$, right $)$ for models M15B11DP2A1H (top) and M15B11UP2A1H (bottom), at various times after their respective explosions commenced (see figure caption and Table 1). All four panels are on a large scale of $8000 \mathrm{~km} \times 8000 \mathrm{~km}$. To facilitate comparison, the same colormaps are used for both $P_{\text {gas }}$ and $P_{\text {mag }}$. The stronger equatorial explosion in model M15B11UP2A1H vis à vis model M15B11DP2A1H is obvious, even though model M15B11DP2A1H has also started to expand laterally and at the times depicted the shock is approaching a radius of $\sim 1000 \mathrm{~km}$ in that direction. It is clear from the right panels that the magnetic stresses are large only interior to the shock, even if it is elongated. This follows quite naturally from the facts that 1) winding is more significant at small radii, 2) the shock flux-compresses accreted field, and 3) as it emerges the jet stretches the large toroidal component generated in the interior and, thereby, enhances the B-field along the axis at larger radii.

Superposed on each left panel are vectors that represent the hydrodynamic flux at a given point. This is the argument of the divergence term in the momentum equation (including the gravitational term), and we refer to it as the "Bernoulli" flux. It does not include the corresponding magnetic term. This is one component in the jet power density and, quite naturally, is pointing outwards. The vectors on the right panels are the Poynting fluxes, the MHD power densities, and they too point outwards and predominantly along the axes. Note, however, that at later times in model M15B11UP2A1H both the Poynting and Bernoulli fluxes have significant components in the equatorial direction. These vector maps detail the instantaneous driving terms of the jet phenomenon in MHD-driven supernova explosions.

Figures 9 and 10 show close-up views on a scale of $2000 \mathrm{~km} \times 2000 \mathrm{~km}$ of the ratio of $P_{\text {mag }}$ to $P_{\text {gas }}$ for models M15B11DP2A1H and M15B11UP2A1H, respectively, at times $430 \mathrm{~ms}$ and $312 \mathrm{~ms}$ after bounce. In these plots, the vectors are velocity vectors. These figures vividly communicate the fact that explosion along the poles and inflow/accretion at the equator are simultaneous. Note that the largest values of $\frac{P_{\mathrm{mag}}}{P_{\mathrm{gas}}}$ are found along the poles and are of order unity (as is also clearly demonstrated in Figs. 2 and 3). Figure 10 also shows that along the poles the jet experiences oscillations as it emerges. These are partially explained by magnetic sausage/neck instabilities. The periods of these instabilities are only a few milliseconds, and the wavelengths are only $\sim 200 \mathrm{~km}$, but we see them in

as a "magnetic tower." 
all our simulations. What consequences or signatures, if any, these sausage instabilities may have remains to be seen.

Given the fact that in the first many hundreds of milliseconds after bounce equatorial accretion and polar explosion occur simultaneously, it is interesting to compare the mass accretion rates with the mass ejection rates. In Fig. 11. we provide both rates versus time after bounce for models M15B11DP2A1H, M15B11UP2A1H, M15B12DP2A1H, and M15B11DP4A1H. The latter model is the same as model M15B11DP2A1H, but with a slower $P_{0}$ of 4 seconds. Comparing the broken and solid red curves on Fig. 11, we see that the jets generated in model M15B11UP2A1H achieve an $\dot{M}$ that exceeds the accretion $\dot{M}$ soon after the explosion commences. In fact, the rapid turndown of the solid red curve indicates that the equatorial explosion occurs rather early. In contrast, the two black curves depicting the corresponding behavior of model M15B11DP2A1H indicate that after $\sim 0.6$ seconds the accretion and jet $\dot{M}$ s are of comparable and modest magnitudes $\left(\sim 0.1 \mathrm{M}_{\odot} \mathrm{s}^{-1}\right)$. We surmise that the actual behavior for a $P_{0}=2$ seconds may be roughly bracketed by these two behaviors during the first second after bounce.

On Fig. 11, we also show $\dot{M}$ s for models M15B12DP2A1H (very explosive) and M15B11DP4A1H. In fact, the simulation of model M15B12DP2A1H crashed early and we have not restarted it. However, its rapid rise speaks for itself. As our arguments in $\$ 2.3$ suggest, we do not think that this behavior reflects what would actually happen for $P_{0}=2$ seconds, but might reflect what would happen for $P_{0}<1$ seconds. However, the anemic $\dot{M}$ seen in Fig. 11 for model M15B11DP4A1H may indicate that $P_{0}$ s of 4 seconds or more would lead, at best, to weak MHD explosions. As we will show in 6 and Fig. 17, though this model does explode via an MHD-driven jet, its explosion energy in the first second is less than a paltry $10^{49}$ ergs (0.01 Bethe).

The magnetic stresses $(\propto(\nabla \times \vec{B}) \times \vec{B})$ are the agencies of jet propulsion and their distribution and direction explain the underlying dynamics. Figures 12 and 13 depict the entropy fields for models M15B11DP4A1H and M15B11UP2A1H, respectively, at post-bounce times of $444 \mathrm{~ms}$ and $312 \mathrm{~ms}$. The right-hand panels are finer-scale versions of the left-hand panels. Superposed on Figs. 12 and 13 are vector fields of the magnetic stress. As the directions of the vectors make clear, the vertical components of the magnetic stresses along the axis have positive signs. This is what drives the jet. In addition, the fact that the vectors point inward towards the axes demonstrates that the jet is partially confined in the shocked region by hoop stresses. These hoop stresses are even more manifest, if indirectly so, in Fig. 4 .

In Figs. 12 and 13, the polar jet column is revealed to be the highest entropy region (red), but the peak entropies achieved are only 15-20 units (per baryon per Boltzmann's constant). This is much smaller that the corresponding values for the generic neutrino-driven explosions ( 20-40; Kitaura et al. 2006; Dessart et al. 2006a; Buras et al. 2006b) and acoustic explosions ( 50-300; Burrows et al. 2006,2007).

\section{ANGULAR FREQUENCY AND PERIOD EVOLUTION}

As we emphasize throughout this paper, rapid rotation is central to the phenomena we are calculating and describing. Collapse transforms initial rotation rates into spin rates at and after bounce that are 500-1000 times faster (Ott et al. 2006). Furthermore, all reasonable initial conditions result in protoneutron stars with differential rotation and shear (Akiyama et al. 2003). With a $P_{0}$ of 2 seconds, inner core periods of $\sim 2$ ms and outer core periods of $\sim 10-30$ ms arise. Figure 14 provides the evolution of the profile of the angular frequency, $\Omega$, in the equatorial direction for the three models: M15B11DP2A1H (top) M15B11UP2A1H (middle), and M15B11DP4A1H (bottom). The colors and the colorbars indicate the time after bounce represented by each line. We see from Fig. 14 that for models M15B11DP2A1H and M15B11UP2A1H the inner $\sim 30 \mathrm{~km}$ rapidly achieves periods of $<3 \mathrm{~ms}$, and that the PNSs continue to spin up with time. Continuing spin-up is due to continuing accretion, which compresses the core, and to core deleptonization and cooling, which undermines pressure support for the PNS mantle. Note that nowhere in these models does matter achieve Keplerian rotation rates (dashed lines in Fig. 14), though they can get close.

As the bottom panel on Fig. 14 shows, the early post-bounce evolution of the average period of the inner region of model M15B11DP4A1H is qualitatively similar to that of other models. However, the M15B11DP4A1H model explodes weakly, with little mass ejected, and is, therefore, not spun down due to the loss of rotational free energy to counteract the spin-up induced by the contraction of the protoneutron star. Interestingly, of all models, M15B11DP4A1H ends up with the shortest average rotation period in the core, despite being the slowest rotating model initially. We surmise that the reduced initial angular momentum leads to reduced centrifugal support, resulting in more efficient contraction and, therefore, enhanced spin-up. This convergence would explain the similar "final" rotation period in models M15B11DP4A1H and M15B0DP2A1H. The weaker enhancement in magnetic pressure after bounce in model M15B11DP4A1H also leads to a more compact protoneutron star, and, thus, is less slowed-down by expansion than models M15B11DP2A1H and M15B11UP2A1H. Overall, accretion is not what distinguishes these models, since, as we see from Fig. 11, all models follow essentially the same path.

The time histories of the corresponding mean periods in the central regions $\left(>10^{10} \mathrm{~g} \mathrm{~cm}^{-3}\right)$ of the PNS for five models, including model M15B0DP2A1H with no field, model M15B12DP2A1H with the highest (unreasonable?) initial poloidal fields, and model M15B11DP4A1H with a $P_{0}$ of 4 seconds, are portrayed in the left panel of Fig. 15 . We note the variety of behaviors. The extreme model M15B12DP2A1H (dark blue) "starts" at an average period of $\sim 8 \mathrm{~ms}$, but quickly spins down due to large magnetic torques to an average period of $\sim 25 \mathrm{~ms}$ within $\sim 100 \mathrm{~ms}$. This is not only dramatic, but indicates the code's capability to follow the evolution of the entire core and its rotation. The inner region is not excised and magnetic torques are given full rein to operate. Using the formula connecting torque $\left(\frac{d L}{d t}\right)$ with magnetic jet power (Fig. [16),$\dot{E}=\Omega \frac{d L}{d t}$, we can derive $\frac{d L}{d t}$ for this model at the end of the simulation (Table 1): $\frac{d L}{d t} \sim 4 \times 10^{48}$ dyne.cm. Dividing by the PNS mass yields the "specific" spindown torque, $\sim 1.5 \times 10^{15}\left[\mathrm{~cm}^{2} / \mathrm{s}\right] \mathrm{s}^{-1}$. 
The evolution of the average period for baseline models M15B11DP2A1H (black) and M15B11UP2A1H (red) indicates that they spin up after bounce, but also that they evolve slightly differently. This is due in part to their different B-field (and, hence, torque density) distributions, but is also due to their different mass fluxes (Fig. 11) and explosion histories, as well as the arbitrary density cut $\left(>10^{10} \mathrm{~g} \mathrm{~cm}^{-3}\right)$ we apply. Nevertheless, mean periods at the end of these simulations of $\sim 4-6 \mathrm{~ms}$ are instructive, even if they are smaller than the average inferred birth periods of pulsars (see Ott et al. 2006 for a discussion). Model M15B11DP4A1H with the higher initial $P_{0}$ bounces with a slower spin rate, but soon spins up due to inexorable accretion. This model experiences a weak explosion at a later phase and involves an $\dot{M}$ that is low (Fig. 11). Curiously, compression and deleptonization spin this model up to values that are comparable to those achieved by the models with faster initial spins.

The right panel of Fig. 15 depicts the corresponding values of the free energy in differential rotation versus time after bounce. Here, the free energy is calculated by determining the kinetic energy of the PNS, and subtracting from it the kinetic energy for the same object if in solid-body rotation at the same total angular momentum. The density profiles in the two realizations are assumed to be the same, so this procedure is only approximate. Nevertheless, in this manner we obtain a useful measure of the energy available to the MHD jet. As the figure indicates, the free energy available to power the jets in all of the $P_{0}=2$-second models exceeds $3 \times 10^{51}$ ergs ( 3 Bethes). Furthermore, models such as M15B11DP2A1H that explode weakly evolve to increase the free energy available, sometimes to large values that can approach the hypernova regime. It would seem that at $P_{0}=2$ seconds, there is ample energy to power a supernova.

However, model M15B11DP4A1H has significantly less free energy with which to maintain a respectable MHD jet explosion. This energy is even less than the $25 \%$ one might have naively expected and is due to the fact that the feedback of rotation on the dynamics of collapse and accretion results in a less differentially-rotating PNS and in a slightly different density (mass) profile. From this, we conclude that the $\Omega_{0}$-dependence of the MHD jet mechanism may be a stronger function of $\Omega_{0}$ than simple scaling arguments would suggest; the viability of the MHD jet mechanism may be a stiff function of $\Omega_{0}$, and may drop off quite fast with increasing $P_{0}$. This possibility puts a premium on precise (more precise) initial models and advanced simulation capabilities.

\section{JET POWERS AND EXPLOSION ENERGIES}

The most important information from our simulations is the energetics of the explosions. The jets carry kinetic, internal, gravitational, and magnetic energies. The total explosion power can be estimated by summing the corresponding contributions for the ejecta. We integrate the Bernoulli and Poynting fluxes over the jet area at a 500-km radius ( $\times 2$ to account for the bipolar jets) and plot in Fig. 16 the result versus time after bounce for four representative models. In all cases, at $500 \mathrm{~km}$ the hydrodynamic (Bernoulli) power is larger than the Poynting power, even though in the inner regions the roles are reversed.

Figure 16 shows that the extreme model M15B12DP2A1H achieves a total jet power (Bernoulli plus Poynting) in excess of $3 \times 10^{51} \mathrm{ergs} \mathrm{s}^{-1}$ within $\sim 25 \mathrm{~ms}$ of the onset of explosion, $\sim 75 \mathrm{~ms}$ after bounce. It is clearly headed for much higher values above $10^{52} \mathrm{ergs} \mathrm{s}^{-1}$ when the run crashes. Model M15B11UP2A1H achieves a total jet power near $10^{52}$ $\mathrm{ergs} \mathrm{s}^{-1}$ within 0.5 seconds of bounce and evolves only slowly on secular timescales thereafter. Model M15B11DP2A1H boast lower total powers, but still reaches values near $10^{51} \mathrm{ergs} \mathrm{s}^{-1}$ by 0.6 seconds after bounce. On the basis of the arguments in 22.3 , we believe that models M15B11UP2A1H and M15B11DP4A1H bracket the true results for initial core spins of 2 seconds, so that quasi-steady-state jet powers for this $P_{0}$ are easily near (or greater than) $10^{51} \mathrm{ergs} \mathrm{s}^{-1}$. Such powers, and the corresponding free energies plotted in Fig. 15, are well within the supernova regime. The slower rotator, M15B11DP4A1H, explodes, but musters total powers of only $\sim 0.05 \times 10^{51} \mathrm{ergs} \mathrm{s}^{-1}$.

The rapid rise and near flattening of the explosion powers portrayed in Fig. 16 lends credence to our scenario that an MHD jet engine, energetically fed by continuing accretion and PNS settling, and lasting as long as is necessary (perhaps many seconds) to produce a viable and vigorous explosion, is established in the context of rapid rotation. The total accumulated energy versus time after bounce is plotted in Fig. 17, The hierarchy of behaviors seen in Fig. 16 is replicated in this figure. Model M15B11UP2A1H takes only $\sim 0.45$ seconds to achieve an explosion energy above $10^{51}$ ergs. Model M15B11DP2A1H will take longer, but is headed in that direction. As expected, after $\sim 0.4$ seconds model M15B11DP4A1H has achieved an explosion energy of only $\sim 0.005 \times 10^{51}$ ergs and is destined to remain anemic.

Figure 17also portrays the integrated "net gain" (Bethe \& Wilson 1985) due to neutrino heating in the outer mantle. This number gives a sense of the contribution of neutrino heating to the explosive energy budget. We see that neutrinos can contribute some multiple of $10^{50} \mathrm{ergs}$, most of which in model M15B11DP2A1H is used to lift the ejecta out of the deep gravitational well. In these models, neutrinos do not, without the MHD jet, lead to explosion in themselves.

Finally, Fig. 18 portrays the evolution of the maximum speed in the ejecta as a function of time. The maximum is $\sim 30000-55000 \mathrm{~km} \mathrm{~s}^{-1}$. This is high, but not near the speed of light. If relativistic speeds are ever achieved in the context of rapidly rotating core collapse, this must happen after the jet head achieves a distance of $5000 \mathrm{~km}$ and after a post-bounce time of one second, perhaps long after. In fact, from the analysis of our results viewed collectively, we see no reason that relativistic speeds would obtain as long as a protoneutron star remains at the core and does not collapse into a black hole through the requisite extended phase of mass accretion.

\section{DISCUSSION AND CONCLUSIONS}

In this paper, we have presented the first 2D, rotating, multi-group, radiation magnetohydrodynamics (RMHD) simulations of supernova core collapse, bounce, and explosion. These calculations cover the longest stretch of physical time and incorporate the most physics ever attempted for MHD simulations in the supernova context. Moreover, many 
of the features and results we find are new. Our focus has been on the evolution, strength, and role of magnetic stresses and on the creation and propagation of MHD jets in the context of rapid rotation. We find that a quasi-steady state can be quickly established during which a well-collimated MHD jet is maintained by continuous pumping of power from the differentially rotating core. If the initial spin period of the progenitor core is $\sim 2$ seconds, the free energy reservoir in the secularly evolving PNS is more than adequate to power a supernova explosion, and may be enough for a hypernova. We speculate that general relativity (GR), by making the core more compact and thereby leading to more rapid post-bounce spin rates, will amplify the power and energy of the MHD jet beyond what we see in our Newtonian simulations. GR may also enable progenitors with initial spin periods slightly higher than $\sim 2$ seconds to generate MHD jets with supernova potential. Given this, initial core periods up to $\sim 3$ seconds may lead to strong MHD-powered supernova. Including the effects of small-scale dissipation of heat by the MRI (TQB) might relax this period bound somewhat, enabling progenitors with higher initial periods near $4-5$ seconds to launch strong jets with supernova energies. However, we infer from our suite of simulations that the MHD jet phenomenon is a very stiff function of $\Omega_{0}$. Note that, due to the strong gravitational wave bursts that are signatures of the bounce of rapidly rotating cores, the associated supernovae may be centrally relevant to ongoing efforts to detect gravitational waves (Ott et al. 2004,2007).

We find that the jet emerges first along a core around the rotation axis, when the magnetic stresses behind the shock approach the local gas pressures. It is the power density, not the total power, that is important in overcoming the accretion ram and punching through the local accreta. The jet is well collimated by the infalling material and magnetic hoop stresses, and maintains a small opening angle during our simulations. Hence, it does not experience the Blanford-Payne centrifugal effect to any great degree. Furthermore, we see evidence of sausage/pinch instabilities in the emerging jet stream, but otherwise the jet settles into a very steady pattern.

Neutrino heating is sub-dominant in the rapidly rotating models we explored, but can contribute $10-25 \%$ to the final explosion energy. Hence, we surmise that for a range of rapid rotation rates a hybrid magneto-neutrino mechanism is possible. Moreover, and intriguingly, even though as in models M15B11DP4A1H and M15B10DP2A1H the MHD jet might be weak and the supernova explosion might have a different origin (neutrino, acoustic ?), our simulations suggest that the supernova explosion can be followed by a secondary MHD jet event, itself a weak explosion. Such a secondary explosion, a reasonable consequence of modest, but not rapid, rotation, may accompany most supernovae, but due to the anticipated weakness when the rotation rates are low, to date this feature may have gone unnoticed. However, the jet/counter-jet structure seen in Cas A (Hwang et al. 2004) may be such a secondary explosion.

A theme that emerges from our simulations with rapid rotation is that for all cases in which either the "Millisecond magnetar model" (Usov 1992; Thompson, Chang, \& Quataert 2004; Bucciantini et al. 2006; Metzger, Thompson, \& Quataert 2006) or the collapsar model (MacFadyan \& Woosley 1999) of GRBs or X-ray flashes may be relevant, since both these scenarios require rapid rotation, the first manifestation of such rotation will be the emergence of an MHD jet during the earlier PNS phase we have addressed in this paper. This implies, for instance, that the relativistic jets from the later collapsar/black-hole phase must be preceded by a precursor jet of some significance (Dessart et al., in preparation; see also Wheeler, Meier, \& Wilson 2002 and Wang \& Meszaros 2007). This delay could be seconds and is the time it takes the inner core to accrete to the critical, GR-unstable configuration that dynamically gives birth to a black hole. Before the formation of the black hole, there is more than enough spin angular momentum and free energy of differential rotation in the PNS to generate and power a magnetic tower explosion. Such a jet may or may not be more energetic in aggregate than the GRB jet that follows it later, but it won't, if we are correct, contribute to the ultrarelativistic component of stellar death which may be the central feature of GRBs.

We have included in our model suite two simulations, M15B10DP2A1F $\left(180^{\circ}\right)$ and M15B10DP2A1H $\left(90^{\circ}\right)$, which allow us to gauge the effect of rapid rotation on the SASI. We find that rapid rotation tends to mute it; the SASI takes longer to develop and achieves smaller amplitudes when it does. The magnetic fields in these "B10" runs were too small to effect the SASI more than rotation, given $P_{0}=2$ seconds. However, these conclusions should be considered preliminary until 3D simulations with good input physics and sophisticated radiative transfer are performed.

Our simulations and estimates suggest that whatever the initial rotational and magnetic conditions in the progenitor core, the MRI, dynamo action, and rotational winding alone should completely alter the magnitude and multipolarity structure of the pulsar or magnetar B-field that is formed in the supernova context. Even if the spin rate is low, convection and the SASI will drag and twist the flux-frozen field. Mere flux conservation on collapse, what has been the default explanation for pulsar fields for decades, can't be the whole, or, perhaps, even a major part of the story. There is too much dynamics during the PNS/supernova phase not to have left a profound mark on what remains.

That said, it may be that the initial inner core field is amplified by flux freezing on collapse and is then afterwards preserved by the fact that this inner core is not in strong differential motion (Fig. 14). The outer region between $\sim 10$ $\mathrm{km}$ and $\sim 150 \mathrm{~km}$ may then experience all the winding, twisting and MRI or dynamo amplification during a transient supernova phase, after which, with the establishment of solid-body rotation for the whole core, the field, in what is now the outer crust of the young neutron star, assumes more modest values. These may be lower than the values frozen in the inner core, may be higher, or much higher (magnetar?). Furthermore, the field in the outer crust will experience ohmic dissipation on timescales, which might be short (days to years), determined in part by the temperatures there. These temperatures, and their profiles, are determined by the dynamics during the collapse and supernova phases. So, one is prompted to ask: Can there be a transient phase of high B-field/rotation that is responsible for a supernova, that as the rotational shear subsides and the core spins down transitions to canonical pulsar B-field and spin values? Can the high B-fields decay due to ohmic dissipation in the crust, after being generated during the hot cool-down phase? What is the progenitor dependence of all this? What determines whether a canonical pulsar or a magnetar is left? 
Whatever the answers to these questions, they are determined in no small measure during the dynamical supernova phase. And that phase, if the progenitor core is rapidly rotating, involves MHD jets and bipolar explosions.

To summarize, the new features, phenomena, and possibilities we have identified in the context of rapidly rotating RMHD core collapse are:

1. That the presence of the stalled shock changes the evolution to explosion dramatically. The confinement it enforces delays explosion and enables the B-field to grow to explosive conditions;

- That the above also makes the accreting matter responsible for collimating the MHD jet, and that hoop stresses are sub-dominant in this regard.

- And, moveover, that the Blandford-Payne centrifugal phenomenon is suppressed by the accreta, leading to a "magnetic tower" explosion that is little aided by the centrifugal whip effect.

2. The condition on the ratio of the magnetic to gas pressures behind the shock along the rotation axis that leads to jet explosion. When this ratio reaches $\sim 1$, a polar jet can emerge;

3. Estimates of the range of initial spin periods that may be necessary to "ignite" explosions with energies of supernova or hypernova magnitudes;

4. That accretion and the associated advection of rotational kinetic energy into the core during the engine phase can maintain the MHD jet in a quasi-steady state for a very long time ( $\gtrsim 1$ second), in fact for as long as accretion lasts;

5. In the case when MHD jets predominate, the fractional contribution of neutrino heating to the total supernova/hypernova powers and energies;

6. That even though MHD-jet powered explosions always start along the poles, they can later explode in an equatorial skirt as well, halting accretion;

7. That if the initial rotation rates are modest, but not fast enough to dominate the explosion mechanism itself, secondary MHD jet explosions may still accompany supernovae powered by other means;

8. That rotation partially suppresses the SASI, and that its suppressive effect generally trumps that due to MHD stresses, at least in the first hundreds of milliseconds.

The numerical aspects of our simulations that distinguish them from those of the past and that allowed us to identify these new features include:

- 2D, multi-group flux-limited neutrino radiation transport. No previous simulation, except for that of LeBlanc \& Wilson (1970), included even a crude variant of gray transport;

- The capacity to simulate for a long stretch of physical time ( 1 second). This is in contrast to previous simulations, which generally lasted only a few tens of milliseconds;

- The use of a realistic nuclear equation of state (EOS). Almost all previous work employed a gamma-law EOS;

- The use of realistic massive-star progenitor density, electron fraction, and temperature profiles, though the initial B-fields and rotation profiles we employed are to be considered only demonstrational. Many previous simulations used polytropes or simplified initial cores;

- The ability to follow collapse up to and after shock stagnation realistically by incorporating detailed microphysics, including electron capture, and neutrino transport. Most previous MHD models that followed collapse witnessed a prompt explosion that did not allow the stalled shock and post-bounce accretion to play an important role in the build-up of the core fields and in the generation and geometry of the resulting MHD jet.

The major limitations of our simulations are:

- That they are not 3D;

- That they are not general-relativistic;

- That the neutrino transport is not multi-angle. Such a variant of VULCAN/2D exists (Livne et al. 2004), but is rather slow;

- That we don't resolve the MRI. Higher spatial resolution simulations should remedy this problem;

- That we don't know the initial B-field and rotation states of the collapsing progenitor cores. This is a drawback of all previous work as well. 
Theorists now have a menu of choices with which to explode a supernova. Neutrino heating seems to work, if underenergetically, for both the lowest-mass massive stars (Kitaura et al. 2006; Buras et al. 2006b) and accretioninduced collapse (Dessart et al. 2006a). If 3D effects are shown to be important, the neutrino mechanism may work in the generic case. If no other mechanism aborts it earlier, the acoustic mechanism (Burrows et al. 2006,2007) seems to obtain after a relatively long delay. As we have presented here, rapid rotation leads to strong MHD jets and bipolar explosions. In principle, MHD jet explosions with hypernova-scale energies can emerge from rapidly rotating protoneutron stars, without the need to create a rapidly rotating black hole. Whether such explosions from a PNS can have a significant relativistic component is as yet unknown.

How rare are rapid rotators? Woosley \& Heger (2006) suggest that they might be more common among lowmetallicity massive stars. However rare or common they are, it is extremely unlikely that they are absent from the family of exploding massive stars. The multi-D, multi-group RMHD simulations we have describe here are a start along the path towards more realistic simulations of the possible role of magnetic fields in some of Nature's most dramatic events.

We thank Martin Pessah, Todd Thompson and Stan Woosley, for fruitful discussions and their insight. We acknowledge support for this work from the Scientific Discovery through Advanced Computing (SciDAC) program of the DOE, under grant numbers DE-FC02-01ER41184 and DE-FC02-06ER41452, and from the NSF under grant number AST0504947. E.L. thanks the Israel Science Foundation for support under grant \# 805/04, and C.D.O. and J.W.M. thank the Joint Institute for Nuclear Astrophysics (JINA) for support under NSF grant PHY0216783. This research used resources of the National Energy Research Scientific Computing Center, which is supported by the Office of Science of the U.S. Department of Energy under Contract No. DE-AC03-76SF00098. We are happy to acknowledge the National Center for Computational Sciences at Oak Ridge for an allocation of computer time on Jaguar. We thank Don Fisher for his help generating both color stills and movies associated with this work and Jeff Fookson and Neal Lauver of the Steward Computer Support Group for their invaluable help with the local Beowulf cluster Grendel. Movies of some of the simulations presented in this paper are available from the first author upon request.

\section{REFERENCES}

Akiyama, S., Wheeler, J.C., Meier, D.L., \& Lichtenstadt, I. 2003, ApJ, 584, 954

Akiyama, S. \& Wheeler, J.C. 2005, ApJ, 629, 414

Ardeljan, N.V., Bisnovatyi-Kogan, G.S., \& Moiseenko, S.G. 2000, A\&A, 355, 1181,

Ardeljan, N.V., Bisnovatyi-Kogan, G.S., \& Moiseenko, S.G. 2005, MNRAS, 359, 333

Ardeljan, N.V., Bisnovatyi-Kogan, G.S., \& Moiseenko, S.G. 2005, MNRAS, 359, 333

Balbus, S.A. \& Hawley, J.F. 1991, ApJ, 376, 222

Bethe, H. \& Wilson, J. R. 1985, ApJ, 295, 14

Bisnovatyi-Kogan, G. S., Popov, I. P., \& Samokhin, A. A. 1976, Ap\&SS, 41, 287

Blandford, R.D. \& Payne, D.G. 1982, MNRAS, 199, 883

Blondin, J.M., Mezzacappa, A., \& DeMarino, C. 2003, ApJ, 584, 971

Bucciantini, N., Thompson, T.A., Arons, J., Quataert, E., \& Del Zanna, L. 2006, MNRAS, 368, 1717

Buras, R., Rampp, M., Janka, H.-Th., \& Kifonidis, K. 2006a, A\&A, 447, 1049

Buras, R., Janka, H.-Th., Rampp, M., \& Kifonidis, K. 2006b, A\&A, 457, 281

Burrows, A. \& Lattimer, J.M. 1986, ApJ, 307, 178

Burrows, A., Livne, E., Dessart, L., Ott, C.D., \& Murphy, J. 2006, ApJ, 640, 878

Burrows, A., Livne, E., Dessart, L., Ott, C.D., \& Murphy, J. 2007, ApJ, 655, 416

Dessart, L., Burrows, A., Ott, C.D., Livne, E., Yoon, S.-Y., \& Langer, N. 2006a, ApJ, 644, 1063

Dessart, L., Burrows, A., Livne, E., \& Ott, C.D. 2006b, ApJ, 645, 534

Etienne, Z.B., Liu, Y.T., \& Shapiro, S.L. 2006, Phys. Rev. D, 74, 044030

Foglizzo, T., Scheck, L., \& Janka, H.-T. 2006, ApJ, 652, 1436 (astro-ph/0507636)

Hawley, J.F., Gammie, C.F., \& Balbus, S.A. 1996, ApJ, 464, 690

Heger, A., Langer, N., and Woosley, S.E. 2000, ApJ, 528, 368

Heger, A., Woosley, S.E., \& Spruit, H. 2005, ApJ, 626, 350 (astro-ph/0409422)

Hirschi, R., Meynet, G., \& Maeder, A. 2005, Nuclear Physics A, 758 , p. $234-237$

Hirschi, R., Meynet, G., \& Maeder, A. 2004, A\&A, 425, 649
Hubeny, I. \& Burrows, A. 2007, accepted to ApJ(astro-ph/0609049)

Hwang, U. et al. 2004, ApJ, 615, 117

Kitaura, F.S., Janka, H.-Th., \& Hillebrandt, W. 2006, A\&A, 450, 345

Kotake, K., Sawai, H., Yamada, S., \& Sato, K. 2004, ApJ, 608, 391

Lampe, M. 1991, Physics of fluids. B, Plasma physics, 3, 1521

LeBlanc, J.M. \& Wilson, J.R. 1970, ApJ, 161, 541

Livne, E., Burrows, A., Walder, R., Thompson, T.A., and Lichtenstadt, I. 2004, ApJ, 609, 277

Livne, E., Dessart, L., Burrows, A., \& Meakin, C.A. 2007, accepted to ApJ(astro-ph/0702465)

MacFadyen, A.I. \& Woosley, S.E. 1999, ApJ, 524, 262

Masada, Y., Sano, T., \& Shibata, K. 2007, ApJ, 655, 447 (astro-ph/0610023)

Matt, S., Frank, A., \& Blackman, E.G. 2006, ApJ, 647, L45

Mazzali, P.A. et al. 2006, Nature, 442, 1018

Metzger, B., Thompson, T.A., \& Quataert, E. 2006, astro-ph/0608682

Mizuno, Y., Yamada, S., Koide, S., \& Shibata, K. 2004, ApJ, 606, 395

Moiseenko, S.G., Bisnovatyi-Kogan, G.S., \& Ardeljan, N.V. 2006, MNRAS, 370, 501

Nomoto, K., Maeda, K., Tominaga, N., Ohkubo, T., Deng, J., \& Mazzali, P., 2005, Astrophysics and Space Science, 298, 81

Ohnishi, N., Kotake, K., \& Yamada, S. 2005, astro-ph/0509765

Obergaulinger, M., Aloy, M.A., \& Müller, E. 2006, A\&A, 450, 1107

Obergaulinger, M., Aloy, M.A., Dimmelmeier, H., \& Müller, E. 2006, A\&A, 457, 209

Ott, C.D., Burrows, A., Livne, E., \& Walder, R. 2004, ApJ, 600, 834

Ott, C.D., Burrows, A., Dessart, L., \& Livne, E. 2006, ApJ Suppl., 164, 130

Ott, C.D., Dimmelmeier, H., Marek, A., Janka, H.-T., Hawke, I., Zink, B., \& Schnetter, E. 2007, Phys. Rev. Lett. submitted, astro-ph/0609819

Pessah, M.E., \& Psaltis, D. 2005, ApJ, 628, 879

Pessah, M.E., Chan, C., \& Psaltis, D. 2006, MNRAS, 372, 183 (astro-ph/0603178)

Proga, D. 2005, ApJ, 629, 397

Sawai, H., Kotake, K., \& Yamada, S. 2005, ApJ, 631, 446 
Shibata, M., Liu, Y.T., Shapiro, S.L., \& Stephens, B.C. 2006,

Phys. Rev. D, 74, 104026 (astro-ph/0610840)

Symbalisty, E.M.D. 1984, ApJ, 285, 729

Takiwaki, T., Kotake, K., Nagataki, S., \& Sato, K. 2004, ApJ, 616, 1086

Thompson, T.A., Chang, P., \& Quataert, E. 2004, ApJ, 611, 393

Thompson, T.A., Quataert, E., \& Burrows, A. 2005, ApJ, 620, 861 (TQB)

Usov, V.V. 1992, Nature, 357, 472

Uzdensky, D.A. \& MacFadyen, A.I. 2006a, ApJ, 647, 1192

Uzdensky, D.A. \& MacFadyen, A.I. 2006b, astro-ph/0609047

Walder, R., Burrows, A., Ott, C.D., Livne, E., Lichtenstadt, I., \& Jarrah, M. 2005, ApJ, 626, 317

Wang, L., et al. 2002, ApJ, 579, 671
Wang, L., Baade, D., Höflich, P., \& Wheeler, J.C. 2003, ApJ, 592, 457

Wang, X.-Y. \& Meszaros, P. 2007, astro-ph/0702441

Wheeler, J.C., Yi, I., Hflich, ., \& Wang, L. 2000, ApJ, 537, 810

Wheeler, J.C., Meier, D.L., \& Wilson, J.R. 2002, ApJ, 568, 807

Wilson, J.R. 1985, in Numerical Astrophysics, ed. J. Centrella, J. M. LeBlanc, R. L. Bowers, (Boston: Jones \& Bartlett), p. 422

Wilson, J.R., Mathews, G.J., \& Dalhed, H.E. 2005, ApJ, 628, 335

Woosley, S.E. \& Bloom, J.S. 2006, ARA\&A, 44, 507

Woosley, S.E. \& Heger, A. 2006, ApJ, 637, 914

Yamada, S., \& Sawai, H. 2004, ApJ, 608, 907 
TABLE 1

Properties of MODELS

\begin{tabular}{|c|c|c|c|c|c|c|c|c|c|c|c|c|}
\hline Name & $\begin{array}{l}\text { Mass } \\
\mathrm{M}_{\odot}\end{array}$ & $\begin{array}{c}B_{\text {poloidal }} \\
\text { Gauss }\end{array}$ & $\begin{array}{c}\text { Field } \\
\text { Geometry }\end{array}$ & $\begin{array}{c}P_{0} \\
\mathrm{~s}\end{array}$ & $\begin{array}{l}A_{0} \\
\mathrm{~km}\end{array}$ & $\Delta \theta$ & $\begin{array}{l}\mathrm{t}_{\text {explosion }} \\
\mathrm{ms}\end{array}$ & $\begin{array}{c}\mathrm{t}_{\text {end }} \\
\mathrm{ms}\end{array}$ & $\frac{\mathrm{V}_{\max }}{\mathrm{km} \mathrm{s}^{-1}}$ & $\begin{array}{c}E_{\text {explosion }} \\
10^{51} \text { erg }\end{array}$ & $\begin{array}{c}\text { Power } \\
10^{51} \mathrm{erg} \mathrm{s}^{-1}\end{array}$ & $\begin{array}{c}<\mathrm{P}> \\
\mathrm{ms}\end{array}$ \\
\hline M15B0DP2A1H & 15 & 0 & $\mathrm{~N} / \mathrm{A}$ & 2 & 1000 & $90^{\circ}$ & $\ldots$ & 595 & $\ldots$ & $\ldots$ & $\ldots$ & 3.70 \\
\hline M15B10DP2A1H & 15 & $10^{10}$ & Dipole & 2 & 1000 & $90^{\circ}$ & 550 & 944 & 37000 & 0.03 & 0.155 & 3.14 \\
\hline M15B10DP2A1F & 15 & $10^{10}$ & Dipole & 2 & 1000 & $180^{\circ}$ & 550 & 685 & 37000 & 0.03 & 0.118 & 3.18 \\
\hline M15B11DP2A1H & 15 & $10^{11}$ & Dipole & 2 & 1000 & $90^{\circ}$ & 250 & 636 & 50000 & 0.2 & 0.661 & 6.17 \\
\hline M15B11UP2A1H & 15 & $10^{11}$ & Uniform & 2 & 1000 & $90^{\circ}$ & 180 & 585 & 55000 & 2.0 & 6.832 & 3.98 \\
\hline M15B11DP4A1H & 15 & $10^{11}$ & Dipole & 4 & 1000 & $90^{\circ}$ & 170 & 415 & 33000 & 0.005 & 0.050 & 4.21 \\
\hline M15B12DP2A1H & 15 & $10^{12}$ & Dipole & 2 & 1000 & $90^{\circ}$ & 80 & 111 & 36000 & 0.6 & 3.168 & 25.60 \\
\hline
\end{tabular}

Note. - In this table, texplosion is the time after bounce of the onset of explosion. tend is the time after bounce at which the simulation was terminated. $v_{\max }$ is the maximum speed of the ejecta at any time during the simulation. $\mathrm{E}_{\text {explosion }}$ is an approximation to the explosion energy of the ejecta at time $t_{\text {end }}$ and in all cases is still accumulating. "Power" is the engine power being pumped into the exploding ejecta at time $t_{\text {end }}$ and includes the total Bernoulli and Poynting contributions calculated at a radius of $500 \mathrm{~km}$. All models use as the reference structure the $15-\mathrm{M}_{\odot}$ model m15b6 of Heger, Woosley, \& Spruit (2005), but the magnetic field and angular velocity distributions are set according to simple analytical descriptions. The initial magnetic field is uniform along $z$ within $A_{0}$, and then switched to a dipole (D) outside or stays uniform (U) out to the maximum grid radius. The initial toroidal magnetic field component is chosen to be 3 orders of magnitude smaller than the polodial component, $B_{\text {poloidal }}$. The angular velocity is such that $\Omega(r)=\Omega_{0} /\left(1+\left(r / A_{0}\right)^{2}\right)$, where $\Omega_{0}=2 \pi / P_{0}$, and $P_{0}$ is the initial period in the core. $r$ is the cylindrical radius. For all models, the time of bounce is $\sim 190 \mathrm{~ms}$. The average period, $\langle\mathrm{P}\rangle$, at the end of each simulation is computed using a density cut of $10^{10} \mathrm{~g} \mathrm{~cm}^{-3}$ and assuming solid-body rotation, given the total angular momentum of the matter above this cut. For example, with our definitions, model M15B10DP2A1H is a $15 \mathrm{M}_{\odot}$ model with initial polodial field of $10^{10} \mathrm{G}$, a dipolar configuration outside of the inner $1000 \mathrm{~km}$, with an initial period of $2 \mathrm{~s}$ and a rotation law $\Omega(r)$ characterized as above with the parameter $A_{0}=1000 \mathrm{~km}$, with the simulation performed over one hemisphere only ("H", in a $90^{\circ}$ quadrant). (See text for discussion.) 

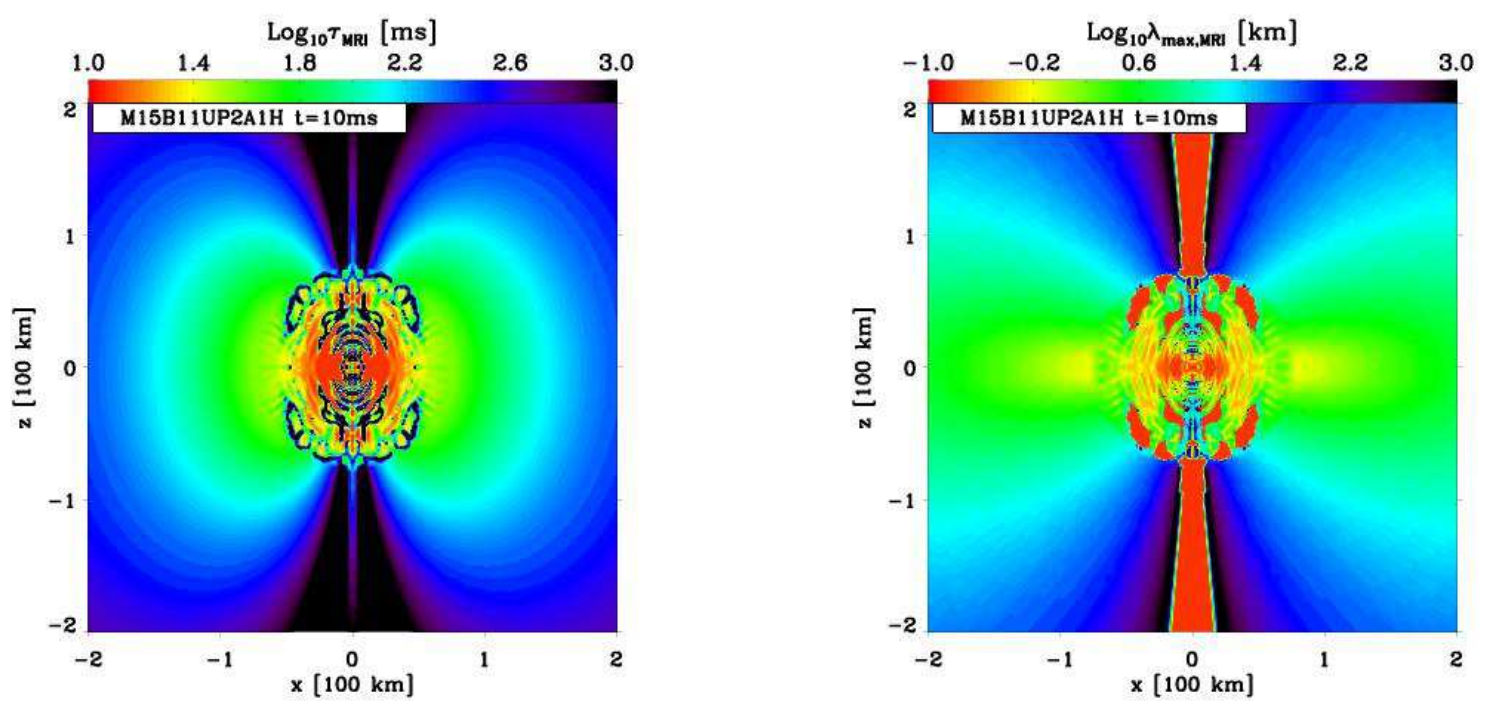

FIG. 1. - Colormap of the growth times (left) and spatial scales (right) for the most unstable mode associated with the magnetorotational instability, obtained using the perturbation formalism of Pessah \& Psaltis (2005) at every interior location in our simulation for model M15B11UP2A1H. (See text for a discussion.) 

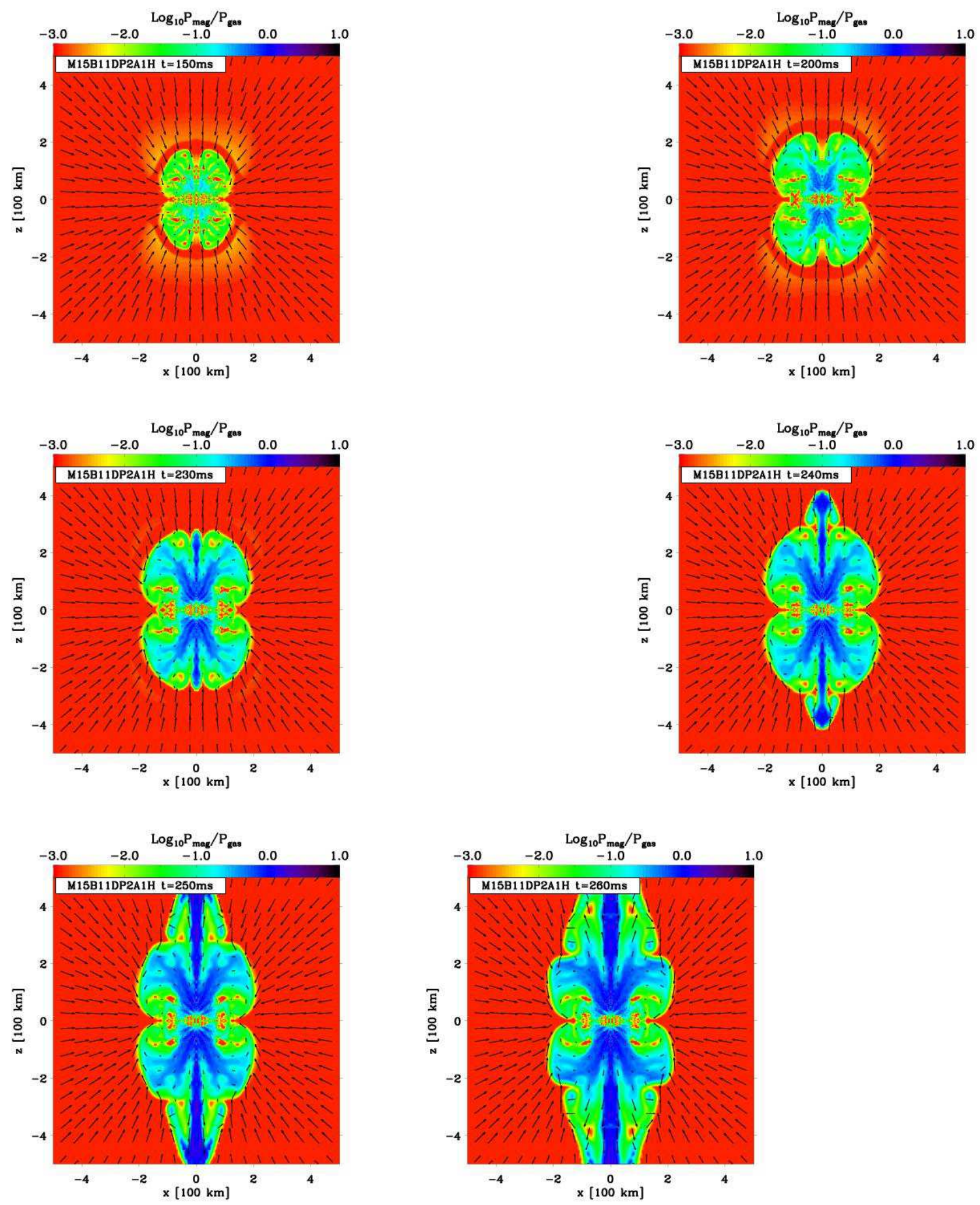

FIG. 2. - Colormap of the ratio of the magnetic pressure to the gas pressure for model M15B11DP2A1H at $150 \mathrm{~ms}$ (top left), $200 \mathrm{~ms}$ (top right), $230 \mathrm{~ms}$ (middle left), $240 \mathrm{~ms}$ (middle right), $250 \mathrm{~ms}$ (bottom left), and $260 \mathrm{~ms}$ (bottom right) after bounce, in the inner $1000 \times 1000 \mathrm{~km}{ }^{2}$ region. This series of six stills shows the approach to and onset of explosion as the magnetic pressure behind the shock becomes more prelevant. We overplot black velocity vectors, with a length saturated at $10000 \mathrm{~km} \mathrm{~s}^{-1}$ that corresponds to $7 \%$ of the width of the display. Note how the magnetic pressure behind the shock (near $\sim 100-200 \mathrm{~km}$ early on) goes from being subdominant to being comparable to the gas pressure. For this model, this happens $\sim 200 \mathrm{~ms}$ after bounce. Notice that though the last three stills are each separated by only $10 \mathrm{~ms}$, once the ratio of the magnetic pressure to the gas pressure near the shock wave is near unity the polar explosion quickly commences. In all our models, the approach of this ratio to unity always signals the onset of a jet explosion, but at different physical times. 

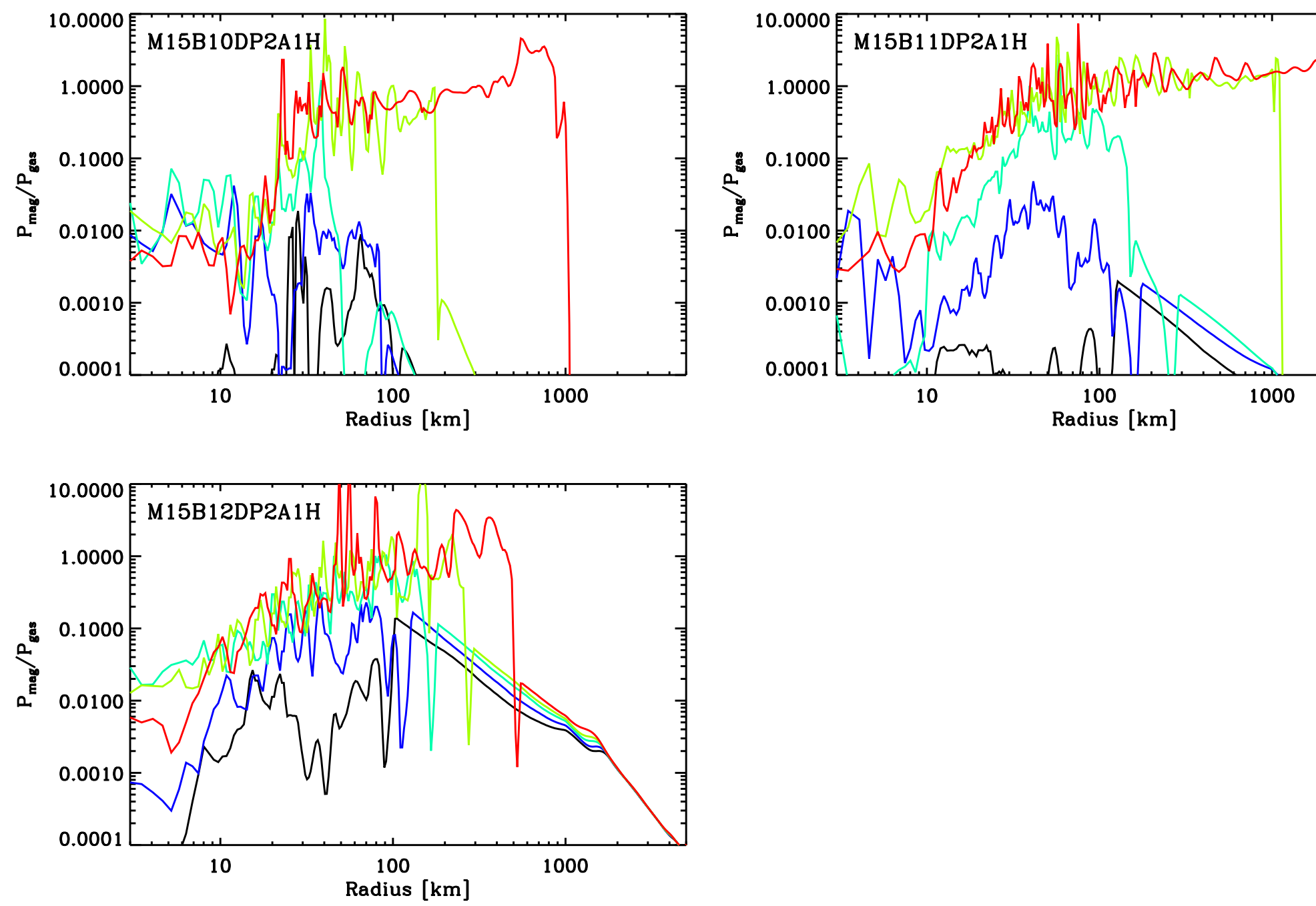

FIG. 3.- Sequence of snapshots of the radial variation along the polar direction of the ratio of magnetic pressure to gas pressure for model M15B10DP2A1H (top left), M15B11DP2A1H (top right), and M15B12DP2A1H (bottom left). The colors corresponds to time, increasing in the order: black, blue, turquoise, green, and red. The turquoise color corresponds to the time when the displayed ratio first exceeds unity, i.e., the time that signals the launching of the jet, and varies from model to model (see Table 1). For model M15B10DP2A1H, the snapshot times are 218, 334, 451, 568, and $685 \mathrm{~ms}$ after bounce. For model M15B11DP2A1H, the snapshot times are 46, 125, 204, 283, and $362 \mathrm{~ms}$ after bounce. For model M15B12DP2A1H, the snapshot times are 21, 35, 49, 63, and $76 \mathrm{~ms}$ after bounce. See text for a discussion. 

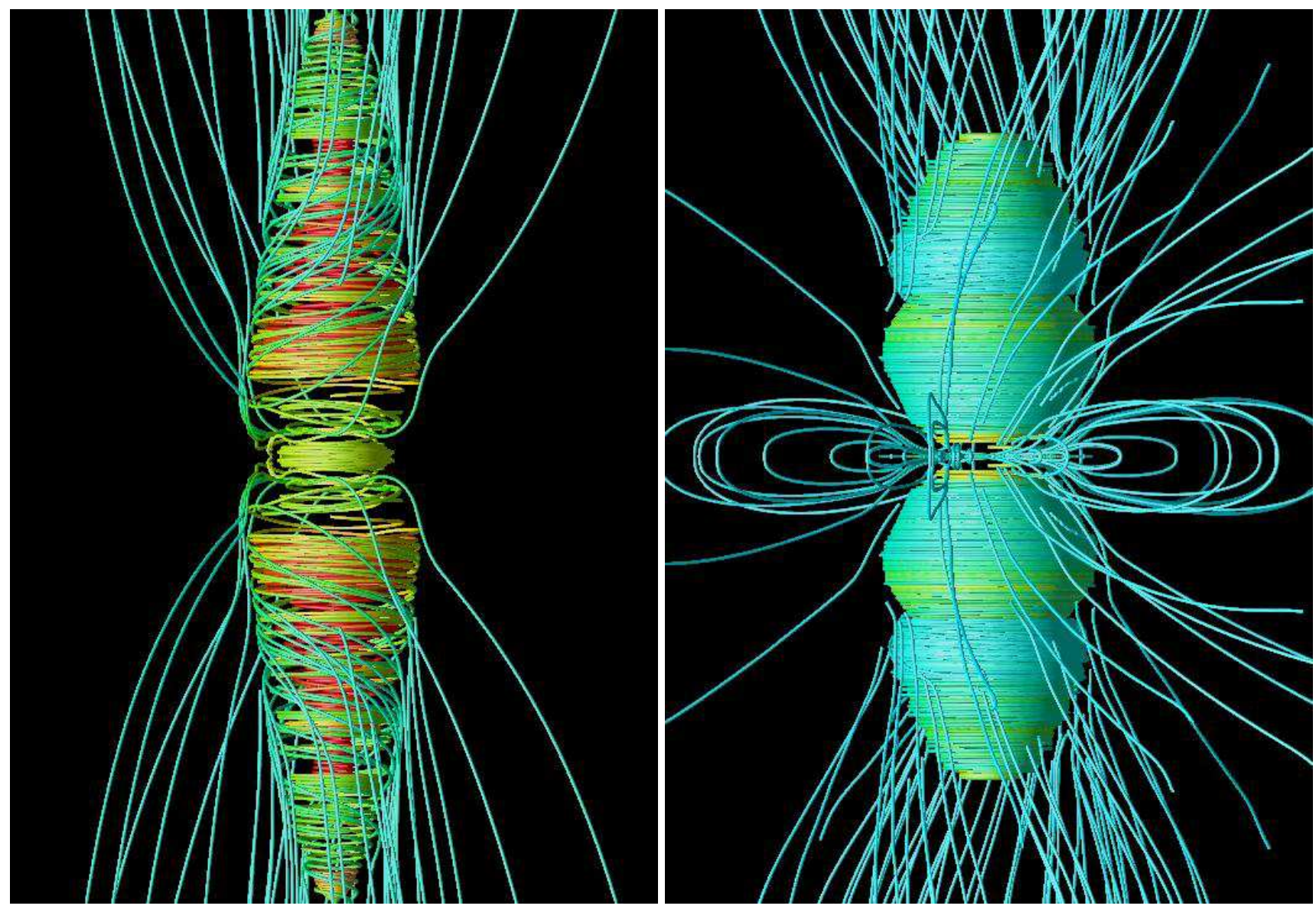

FIG. 4.- Left: Magnetic field lines for model M15B11UP2A1H at $264.5 \mathrm{~ms}$ after bounce. The size of the displayed region is $3000 \times 4000 \mathrm{~km}{ }^{2}$. "Footpoints" for the field lines are randomly distributed in the inner 500-1000 km, with a denser distribution along the polar axis to probe the region of larger magnetic energy where the explosion takes place in our simulations. Hence, the crowding of field lines does not correspond directly and accurately to regions of larger magnetic fields. Right: Same as on the left, but for model M15B10DP2A1H at 855.5 ms after bounce and on a scale of $6000 \mathrm{~km} \times 8000 \mathrm{~km}$. Notice how much more tightly the B-field is wound. (See text for a discussion.) 

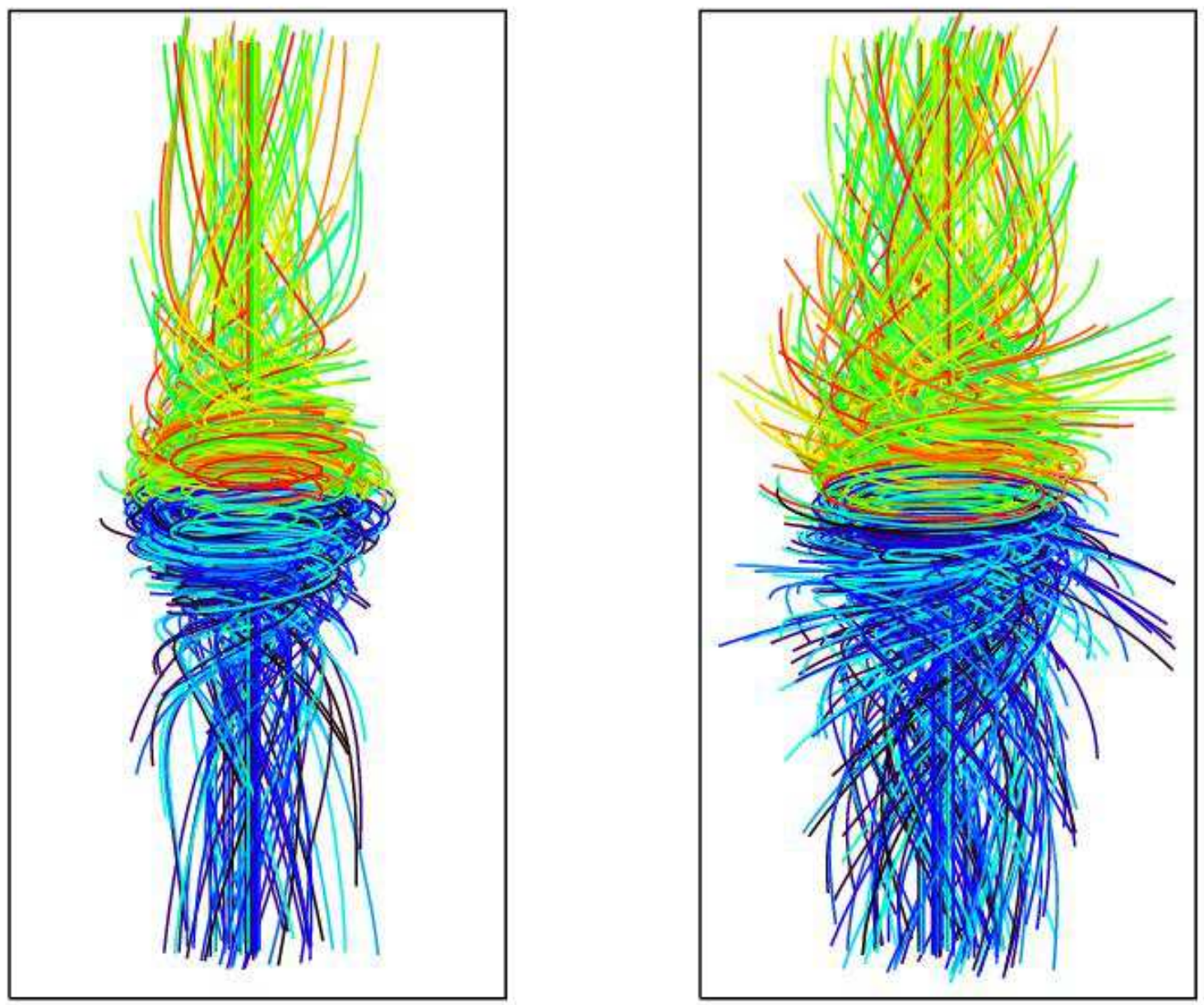

FIG. 5. - Streaklines of marker particles covering the $200 \mathrm{~ms}$ prior to the end of the simulation for each model M15B11DP2A1H (left) and M15B11UP2A1H (right). Markers are originally disributed uniformly with radius, using 30 shells between 50 and $200 \mathrm{~km}$, as well as with angle, using 30 angular beams between the rotation axis and the equatorial direction. The marker azimuth is randomly chosen between $0^{\circ}$ and $360^{\circ}$. In its wanderings, if a marker moves closer than $50 \mathrm{~km}$ from the neutron-star center, we replace it with a new marker located within a cone, along the rotation axis, with a $10^{\circ}$ opening angle and randomly distributed in radius between 50 and $500 \mathrm{~km}$. Here, we show the streaklines for 300 of the 900 particles followed, the displayed region extending out to $500 \mathrm{~km}$ away from the rotation axis, and $1000 \mathrm{~km}$ from the equatorial plane. The viewing angle is $30^{\circ}$ with respect to the equatorial plane, with a color coding randomly allocated, although using warm (cool) colors for the northern (southern) hemisphere. 

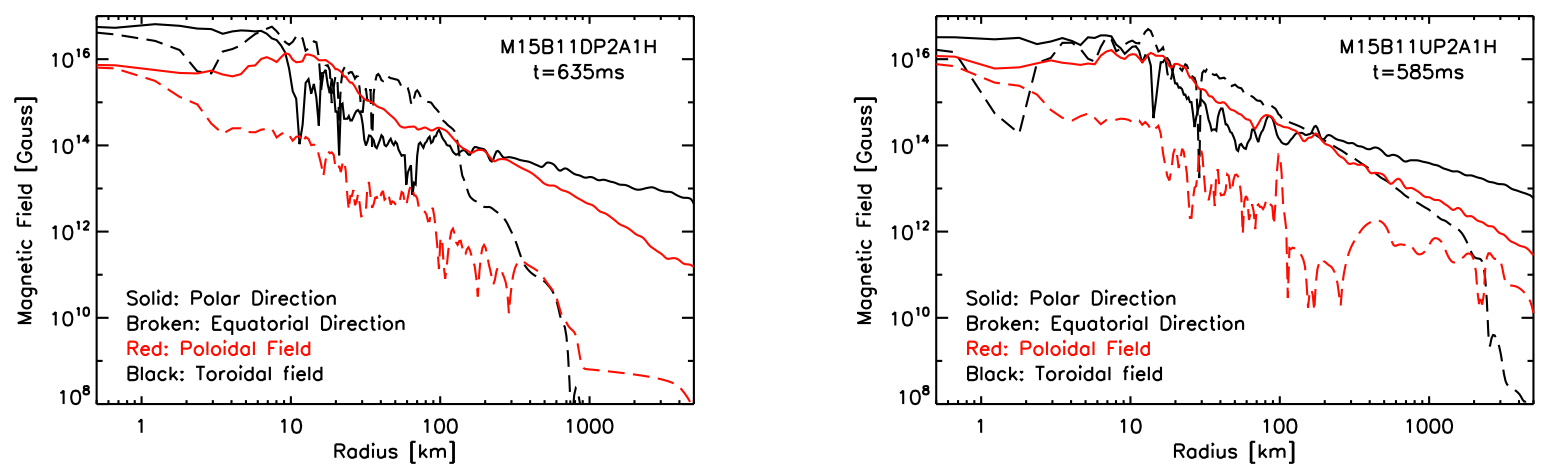

FIG. 6. - Radial slices at the end of each simulation for models M15B11DP2A1H (left) (at 635 ms after bounce) and M15B11UP2A1H (right) (at $585 \mathrm{~ms}$ after bounce) of the magnitude of the toroidal (black) and poloidal (red) components of the magnetic field, along the polar (solid) and equatorial (broken) directions. 

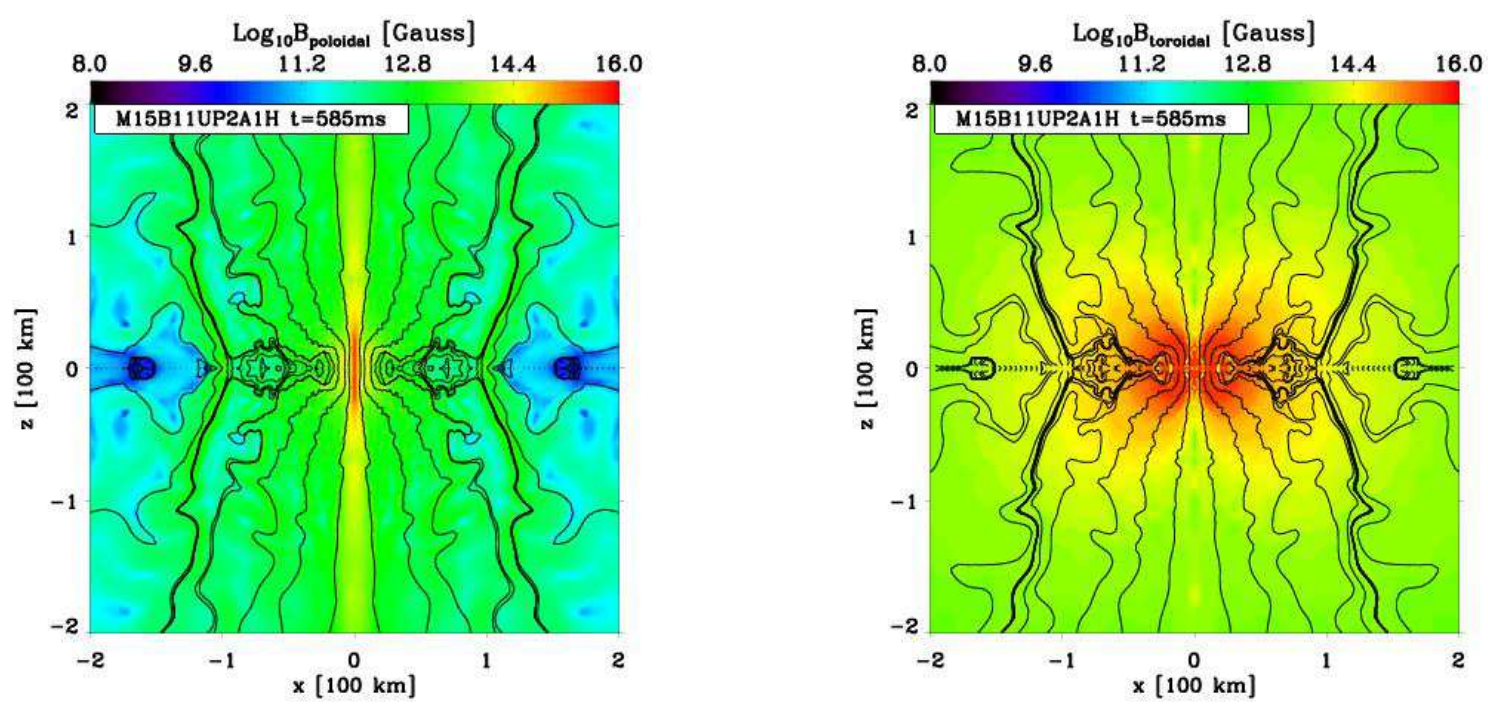

FIG. 7.- Colormap of the magnitude of the poloidal (left panel) and the toroidal (right panel) components of the magnetic field for model M15B11UP2A1H at $585 \mathrm{~ms}$ after bounce out to $200 \mathrm{~km}$. We also overplot poloidal field lines every $4 \mathrm{~km}$, starting along the equator within $200 \mathrm{~km}$ of the polar axis. 

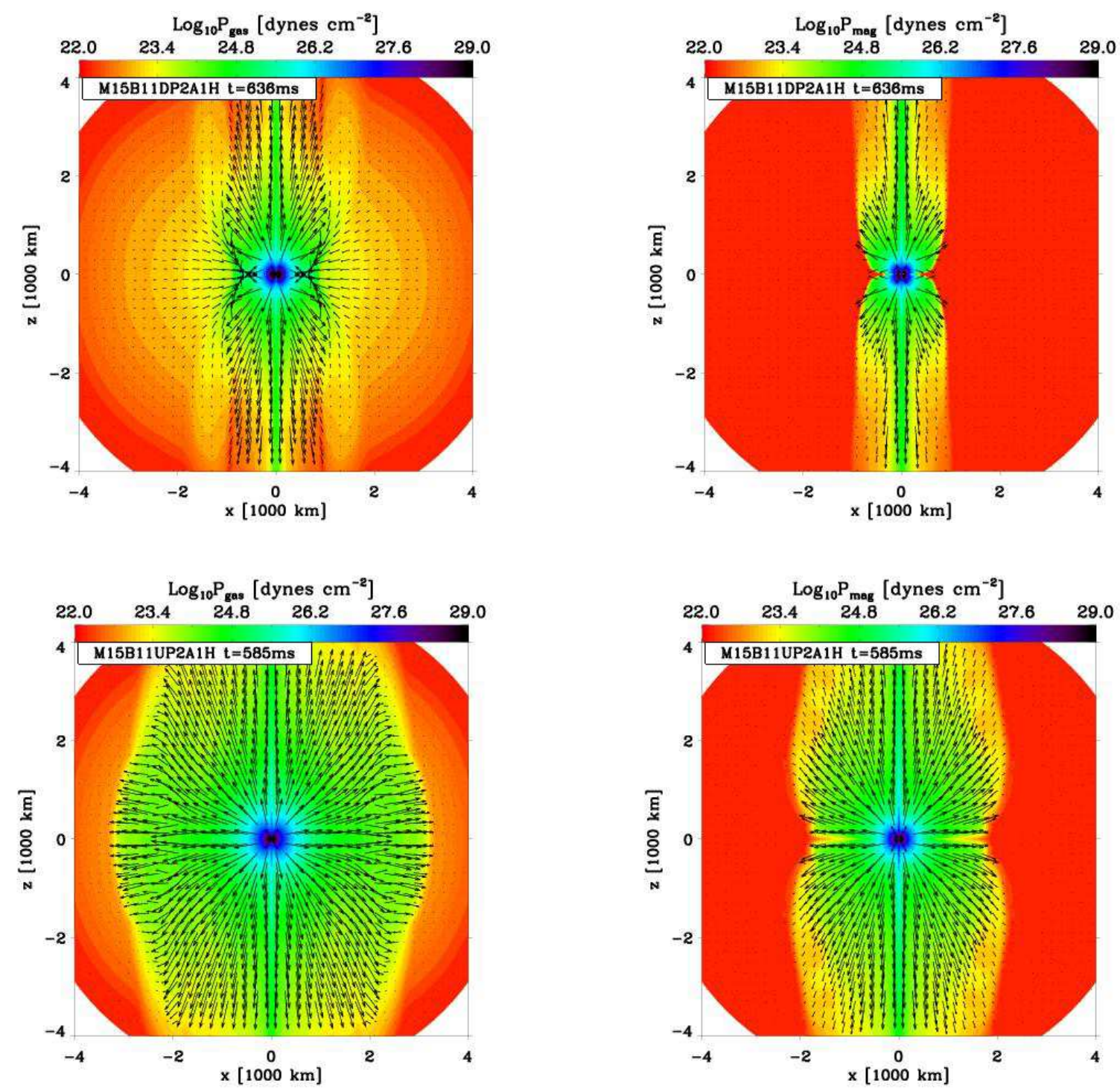

FIG. 8.- Top Left: Colormap of the gas pressure for model M15B11DP2A1H, at $636 \mathrm{~ms}$ after bounce. We also overplot "Bernoulli" vectors in black, corresponding to $\rho v_{R} \times\left(e_{\mathrm{th}}+e_{\mathrm{kin}}+P / \rho-G M_{\mathrm{PNS}} / R\right)$. This choice does not account for contributions from the magnetic field. The vector length is saturated at $10^{33} \mathrm{erg} \mathrm{cm}^{-2} \mathrm{~s}^{-1}$ and corresponds to $15 \%$ of the side length of the display. Top Right: Colormap of the magnetic pressure, at $636 \mathrm{~ms}$ after bounce, including the toroidal and the (sub-dominant) poloidal components. We overplot vectors for the Poynting flux, with the same ratio between vector length and vector magnitude as in the left panel. The Poynting flux has the following standard definition: $\vec{S}=c / 4 \pi(\vec{E} \times \vec{B})$, and measures the rate of electromagnetic energy flow, here on this scale of comparable magnitude to the Bernoulli flux (though lower), shown in the left panel. Bottom Left and Right: Same as above, but for model M15B11UP2A1H at $585 \mathrm{~ms}$. (See text for discussion.) 


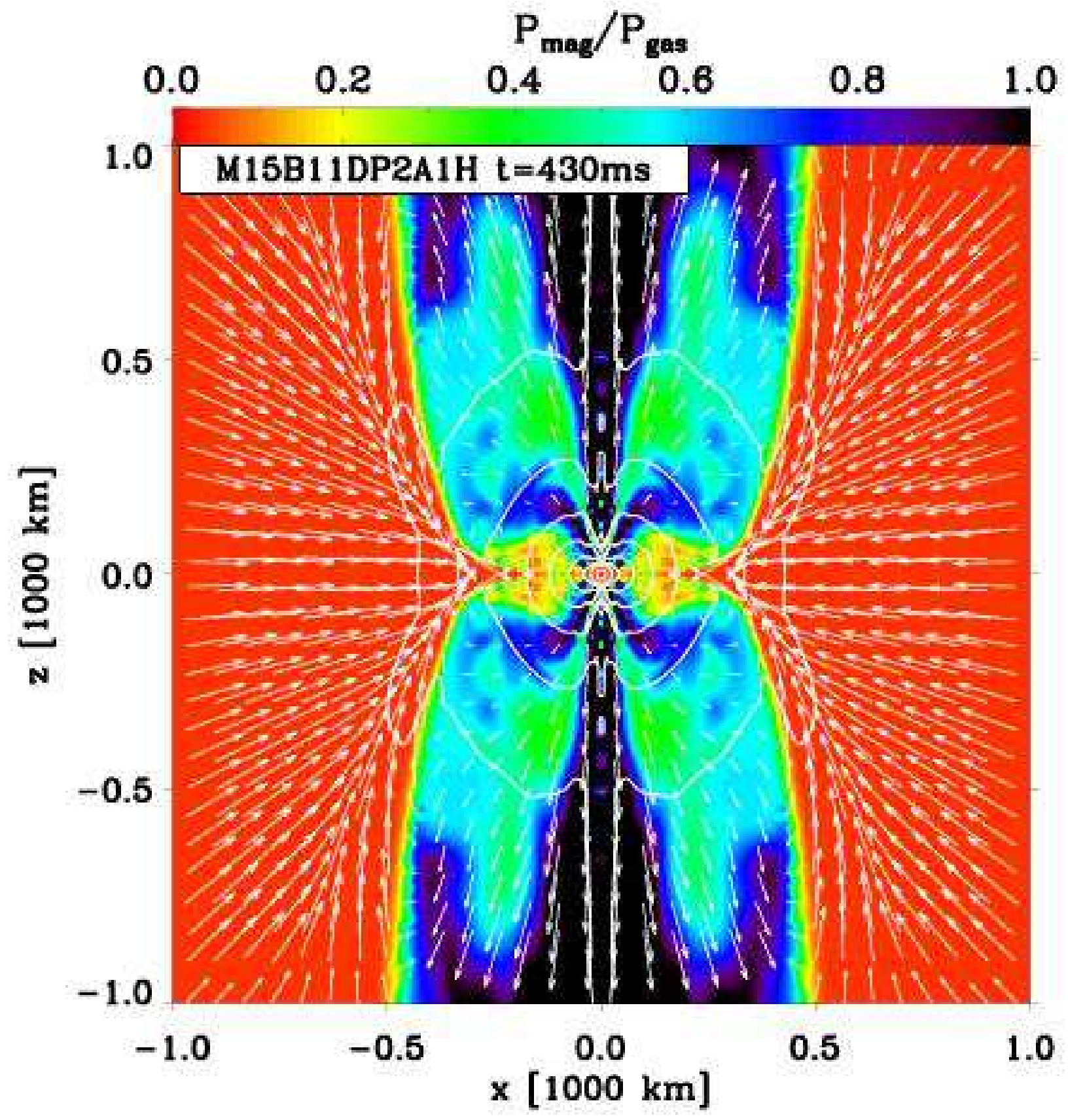

FiG. 9.- Colormap for model M15B11DP2A1H at $430 \mathrm{~ms}$ after bounce of the ratio of magnetic to gas pressure, overplotted with white isodensity contours (every decade downward from $10^{14} \mathrm{~g} \mathrm{~cm}^{-3}$ ) and velocity vectors (length saturated to $15 \%$ of the width of the figure and corresponding to a velocity of $10000 \mathrm{~km} \mathrm{~s}^{-1}$ ). 


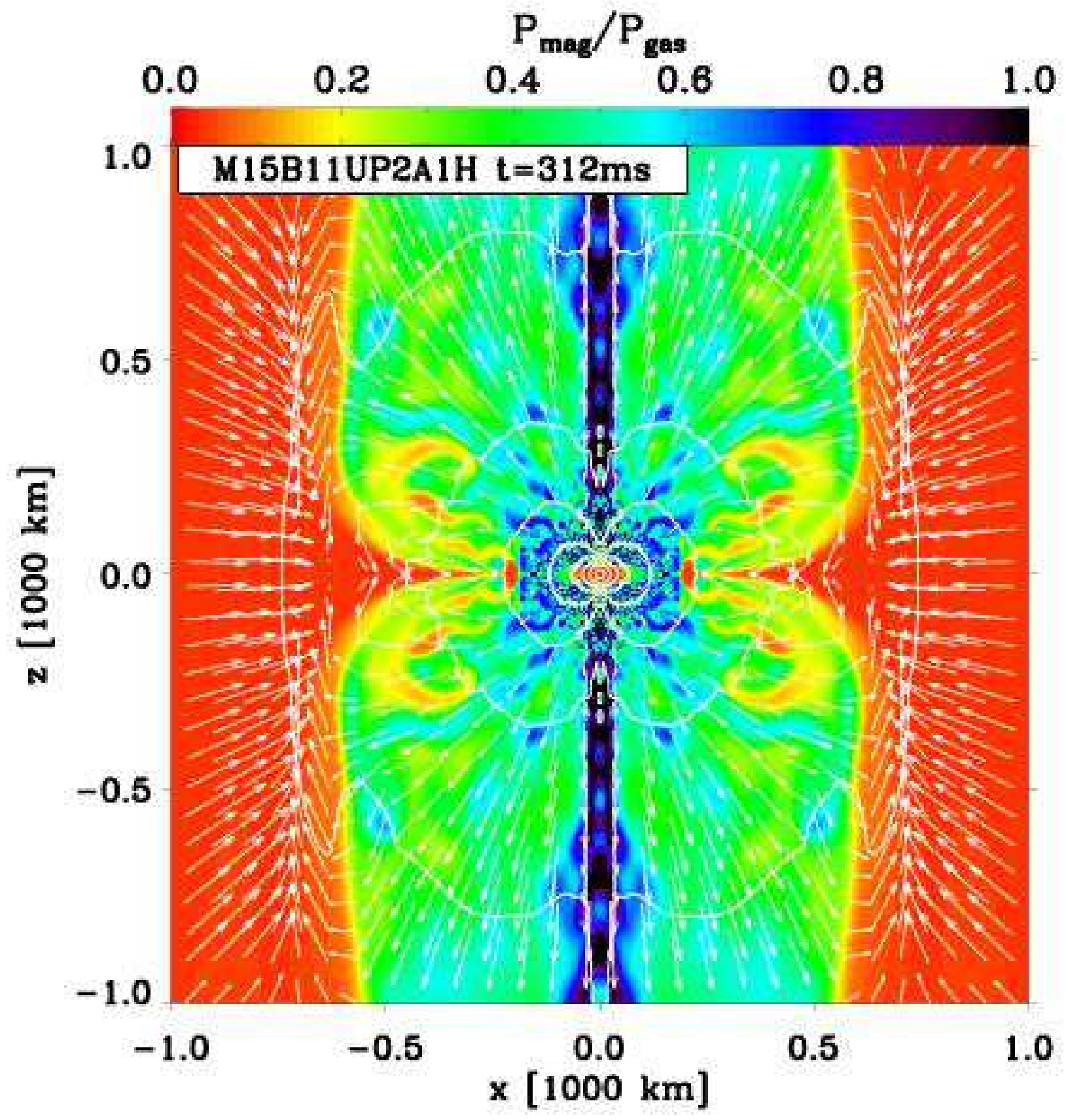

FIG. 10. - Same as Fig. 9] but for model M15B11UP2A1H at $312 \mathrm{~ms}$ after bounce. 


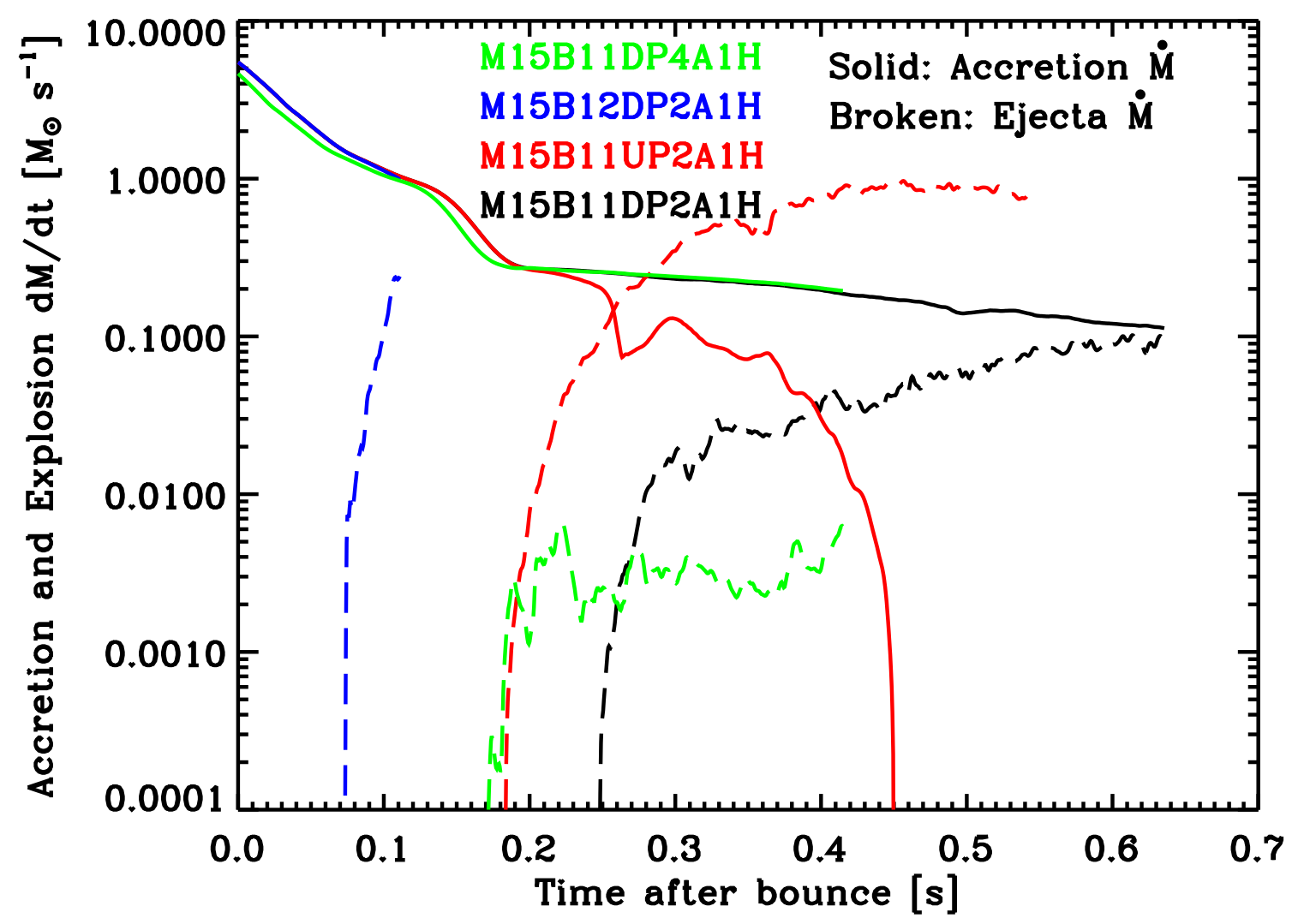

FIG. 11. - Time evolution of the instantaneous integrated mass flux accreting (solid) or outflowing (broken) through a shell at a radius of $500 \mathrm{~km}$, for four representative models. The mass flux for model M15B0DP2A1H (not shown) is very similar to that for model M15B11DP2A1H. 

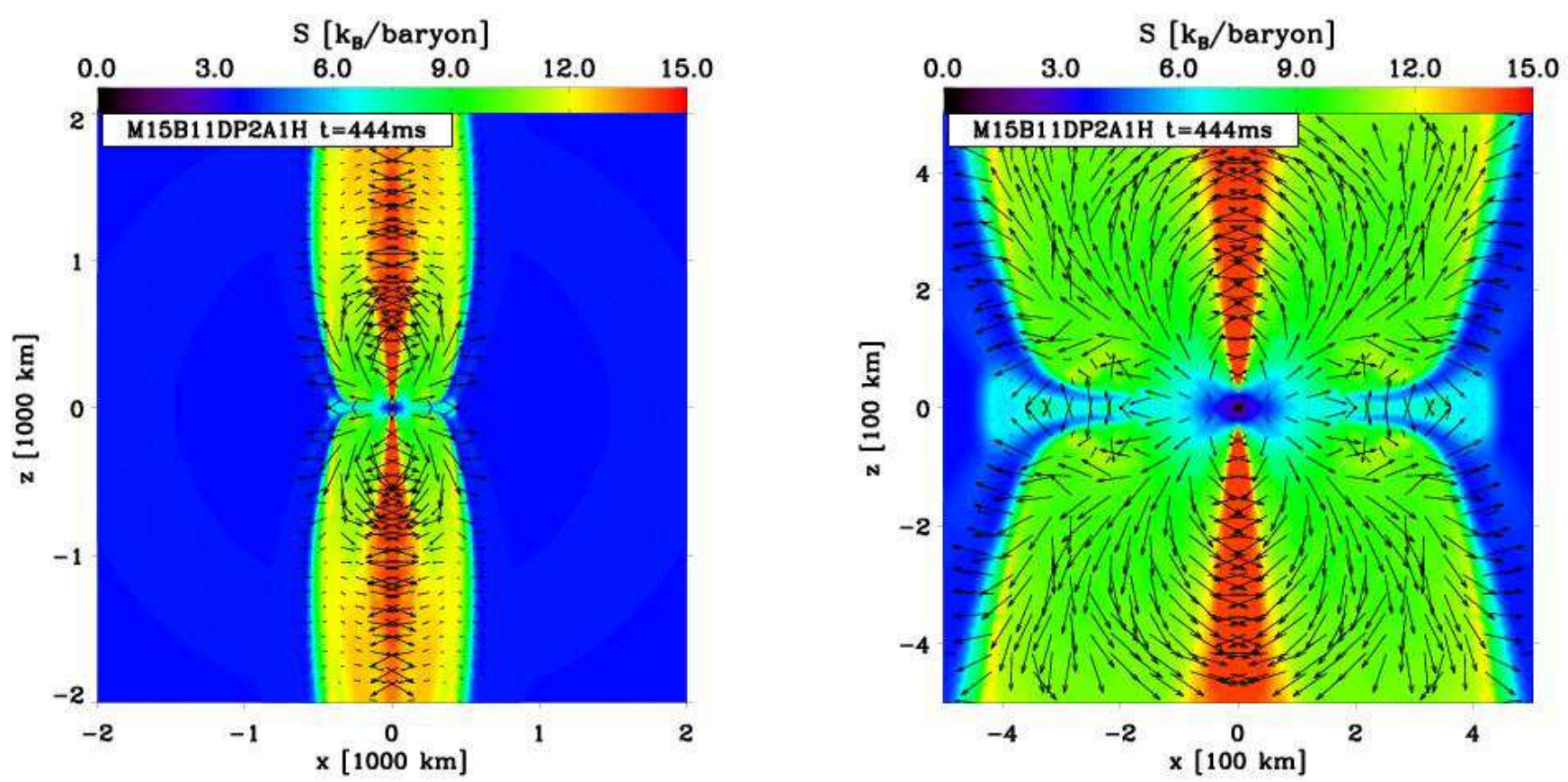

Fig. 12.- On the left-hand-side is a colormap of the entropy at $444 \mathrm{~ms}$ after bounce for model M15B11DP2A1H on a $4000 \mathrm{~km} \times 4000$ $\mathrm{km}$ scale. We overplot the $(\nabla \times \vec{B}) \times \vec{B}$ vector field, with a length of $15 \%$ of the width of the display corresponding to a saturation value of $10^{18} \mathrm{~g} \mathrm{~cm}^{-2} \mathrm{~s}^{-2}$. This term enters the momentum equation and thus represents the acceleration due to the magnetic field, revealing here, in particular, the role of hoop stresses in confining the jet as it moves to large distances. The right panel is the same as the left panel, but for the inner $1000 \mathrm{~km} \times 1000 \mathrm{~km}$ region. 

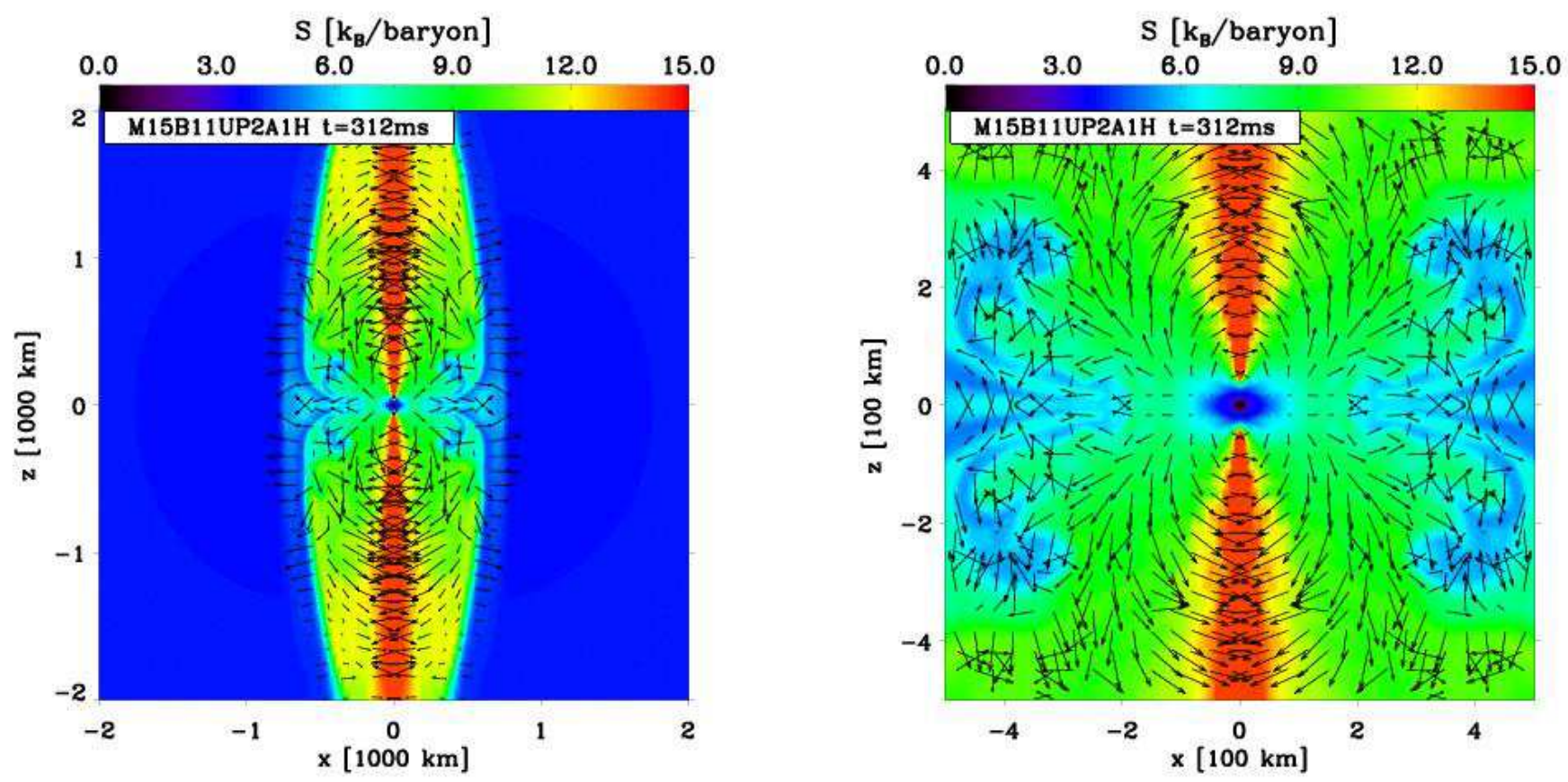

FIG. 13. - Same as for Fig. 12, but for model M15B11UP2A1H, at $312 \mathrm{~ms}$ after bounce. We use the same length-magnitude relationship for the vectors. 

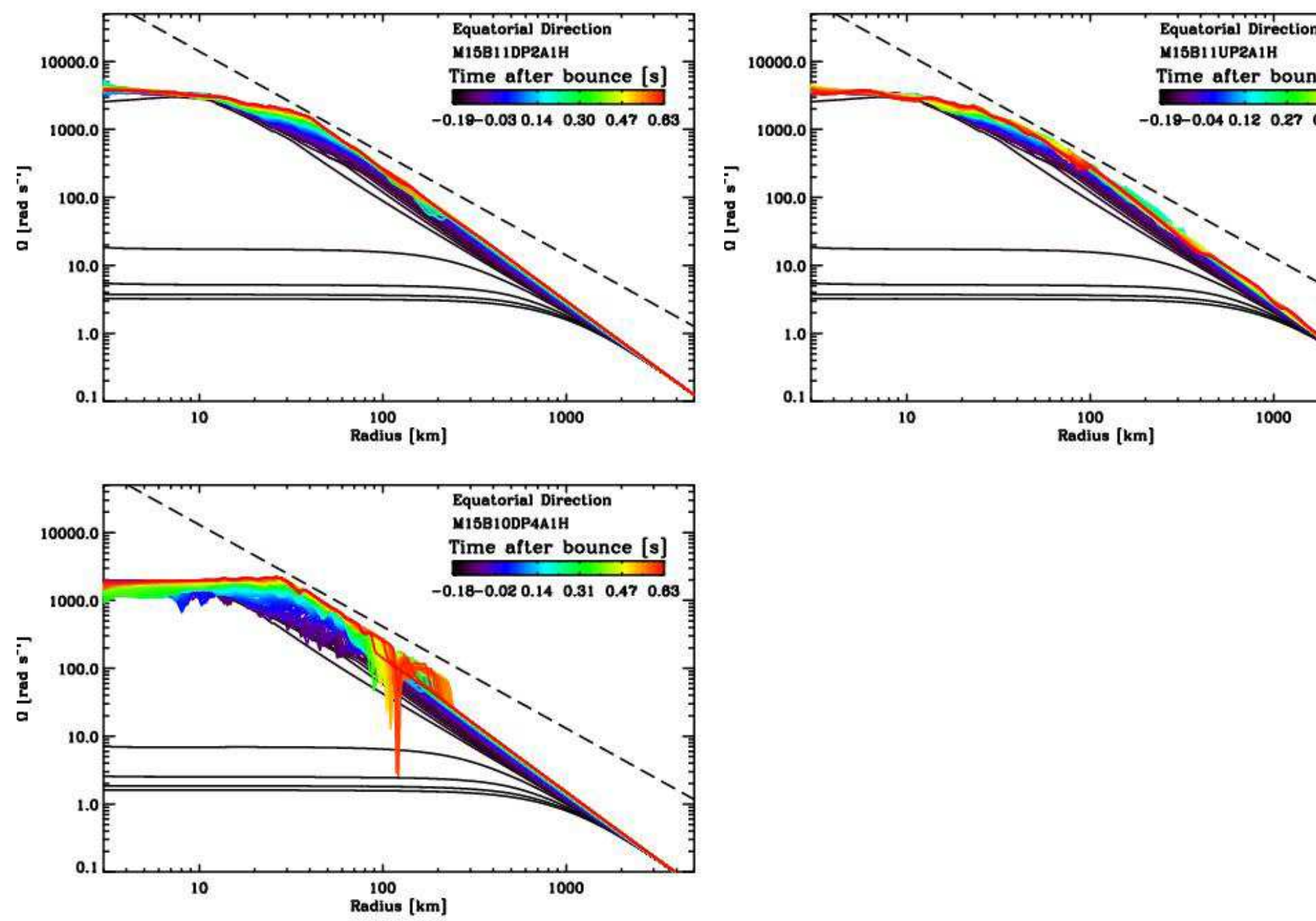

FIG. 14.- The time evolution (see colorbar) of the equatorial angular velocity for models M15B11DP2A1H (top left), M15B11UP2A1H (top right), and M15B11DP4A1H (bottom). For comparison, we also overplot in each panel, as a dashed line, the local Keplerian velocity for the last time. Notice the solid-body rotation inside a radius of $\sim 20-30 \mathrm{~km}$, with a monotonic spin-up at all radii as time progresses. For model M15B11DP4A1H, the angular velocity evolution reflects primarily the collapse of the progenitor envelope, with lesser effects due to the weak explosion and modest mass loss. See text for a discussion. 

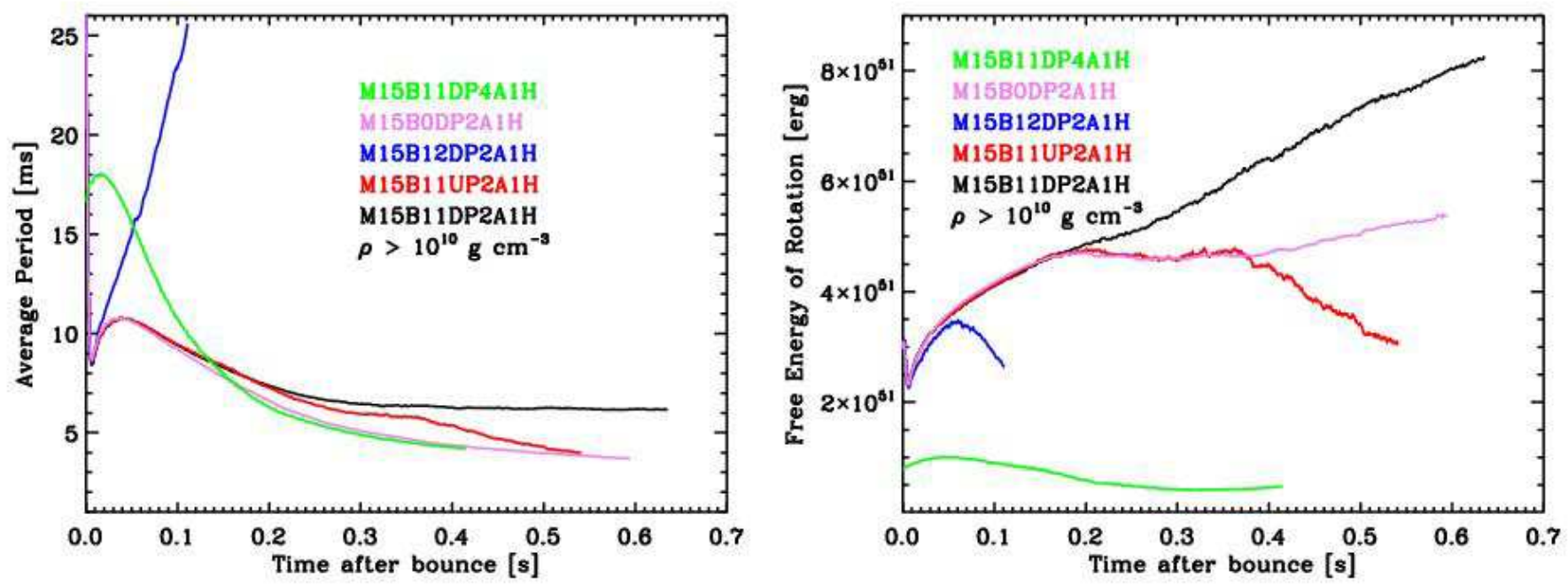

FIG. 15. - Left: Time evolution for models of the average period, assuming solid body rotation for the corresponding cumulative angular momentum, for the region interior to the iso-density contour corresponding to $10^{10} \mathrm{~g} \mathrm{~cm}^{-3}$. Right: Time evolution for models of the free energy of rotation interior to the iso-density contour corresponding to $10^{10} \mathrm{~g} \mathrm{~cm}^{-3}$. (See text for discussion.) 


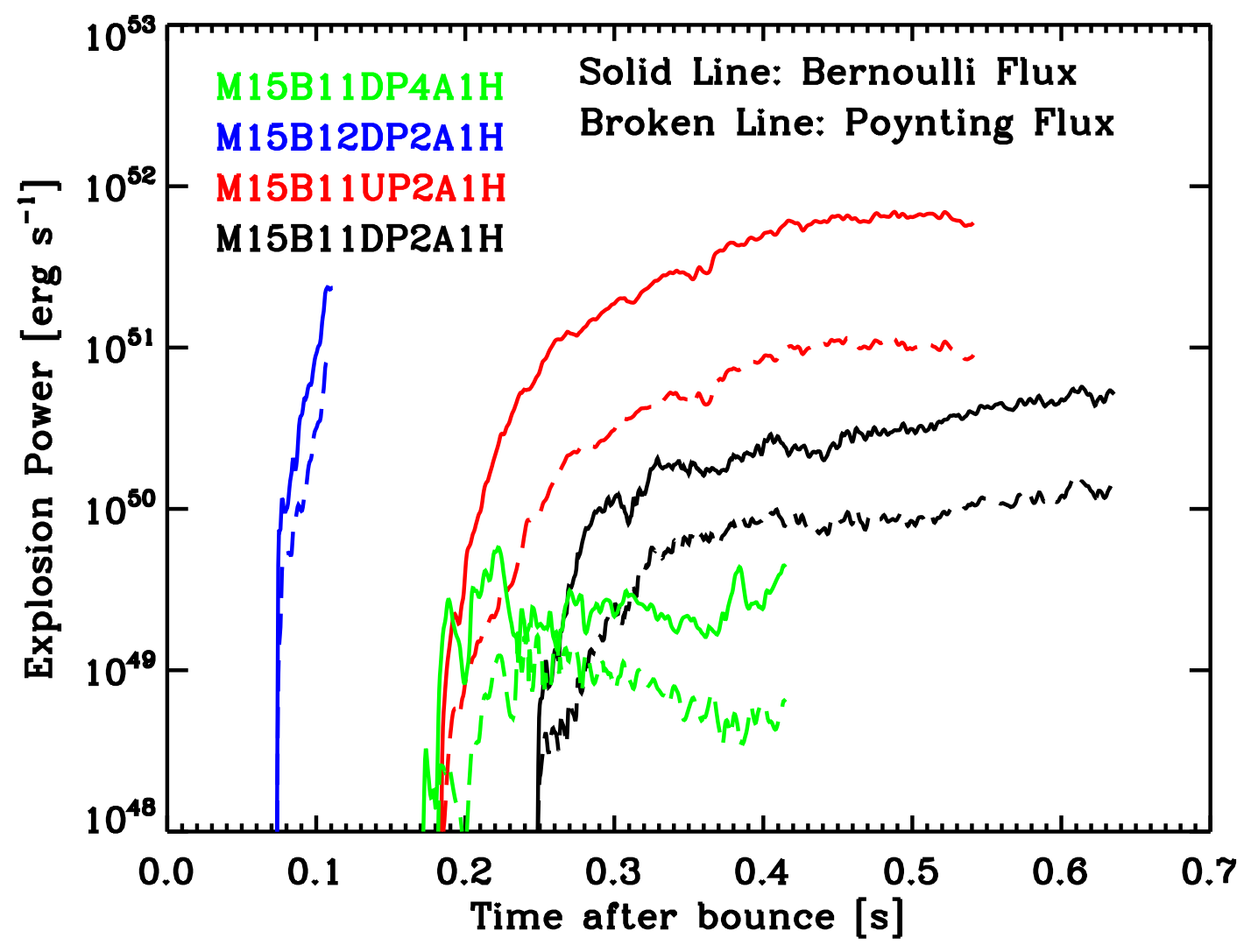

FIG. 16. - Time evolution of the hydrodynamic/Bernoulli (solid line) and Poynting (broken line) powers in the ejecta, integrated over a radial shell at $500 \mathrm{~km}$, for four of the models simulated. 


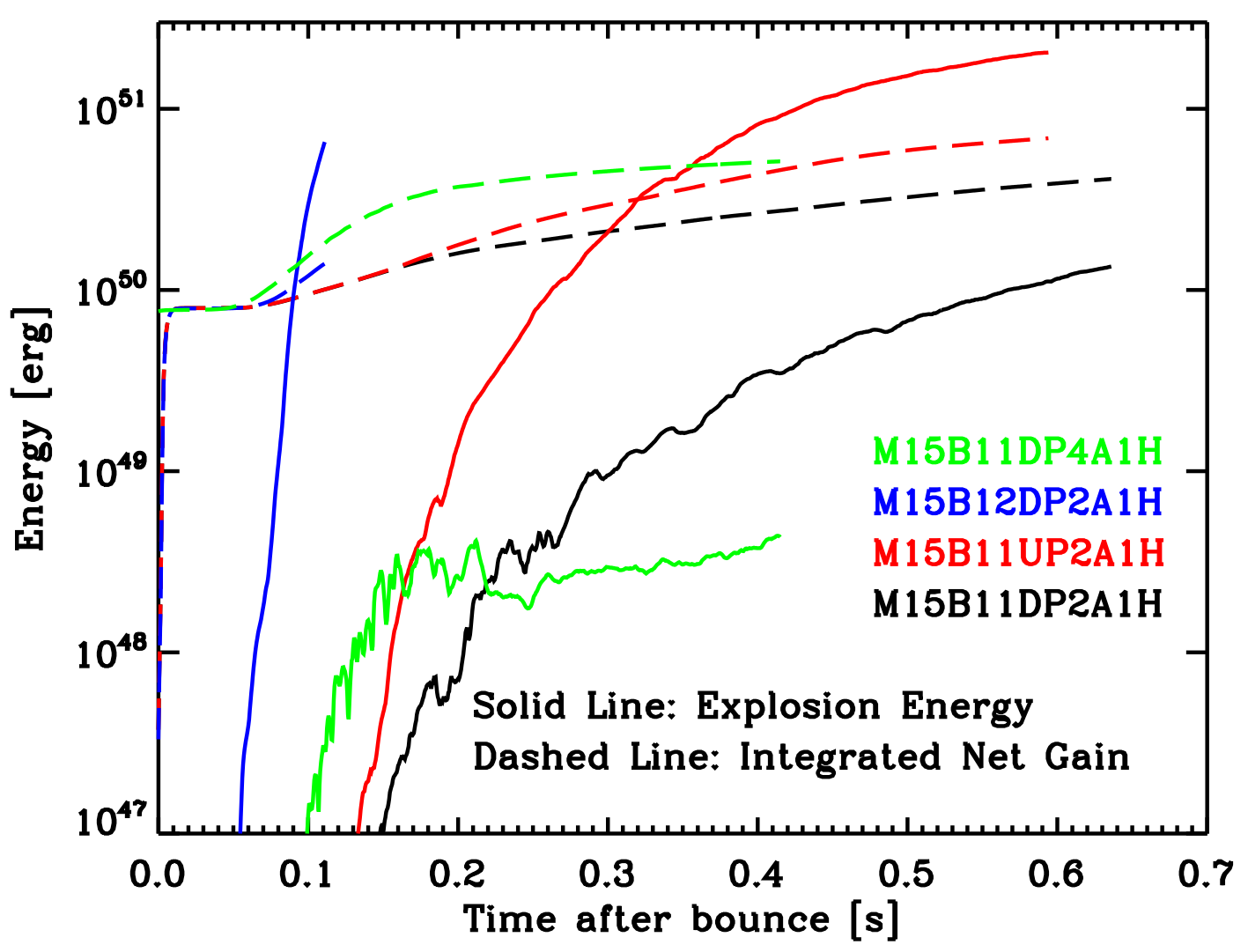

FIG. 17. - Time evolution of the explosion energy (solid line) and the net integrated neutrino gain (dashed) due to deposition outside the high-density regions limited by the $10^{10} \mathrm{~g} \mathrm{~cm}^{-3}$ contour, for four models simulated in this study. (See text for discussion.) 


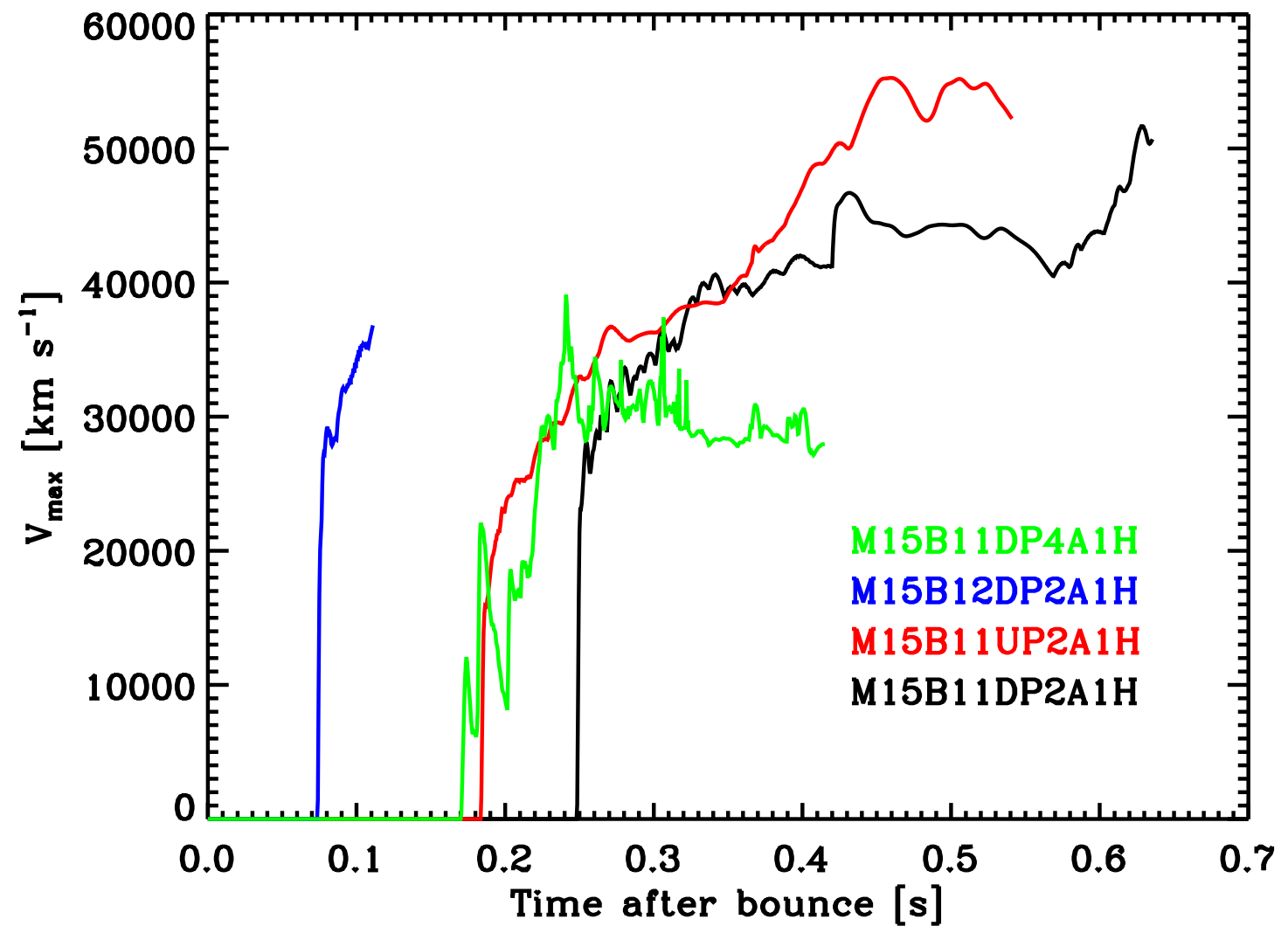

FIG. 18. - Time evolution for four of the models we simulated in this study of the maximum (outward-oriented) velocity along the polar axis and outside a radius of $500 \mathrm{~km}$. 